\title{
Die distale Radiusfraktur
}

\author{
A. Wichelhaus, G. Gradl, T. Mittlmeier
}

Unfall- und Wiederherstellungschirurgie, Chirurgische Universitätsklinik Rostock

Die distale Radiusfraktur ist die häufigste Fraktur des Menschen und verursacht einen erheblichen Arbeitsanfall in chirurgischen Notaufnahmen.

Vor 20 Jahren wurde ein Großteil der distalen Radiusfrakturen konservativ therapiert, heute jedoch setzt sich mehr und mehr die palmare winkelstabile Plattenosteosynthese durch. Jüngste Studien zeigen gute Behandlungsresultate bei jedoch nicht unerheblicher Komplikationsrate. Hauptprobleme bleiben die langfristige Stabilisierung der dorsalen Trümmerzone, Sehnenkomplikationen und die Reposition dorsaler Gelenkfragmente. Hierfür kann der dorsale Zugang notwendig sein. Der Fixateur externe und die Kirschner-Draht-
Osteosynthese sind in der Literatur weiterhin Standardverfahren. Begleitverletzungen des Carpus und des Bandapparats sollten nicht übersehen werden und bedürfen mitunter einer besonderen Behandlung. Der komplexen Frakturgeometrie instabiler Radiusfrakturen kann mit einem Verfahren allein und mit einem operativen Zugangsweg oftmals nicht Rechnung getragen werden.

Wir möchten mit diesem Beitrag einen Überblick über die verschiedenen Behandlungsmöglichkeiten geben und Hilfe leisten zur Therapieplanung, die auf den Frakturtyp und den Patienten individuell angepasst sein sollte.

\section{Epidemiologie und Ätiologie}

Die distale Radiusfraktur entsteht in der Regel durch einen Sturz auf die abfangende Hand. Betroffen sind einerseits junge, sportlich aktive und risikobereite Menschen, die durch ein Trauma mit erheblicher Gewalteinwirkung Radiusfrakturen erleiden. Viel mehr aber sind ältere Patienten, zumeist Frauen mit Osteoporose betroffen, bei denen durch sogenannte „low energy falls“ eine Fraktur des distalen Radius entsteht.

In Deutschland werden pro Jahr etwa 200000 distale Radiusfrakturen behandelt. In epidemiologischen Studien wird das Risiko für eine Fraktur des Radius mit 1:1000 für Menschen unter 65 Jahre beziffert. Dieses Risiko steigt bei über 85-Jährigen auf 12:1000. Frauen sind etwa $4 \times$ häufiger betroffen als Männer.

Die distale Radiusfraktur wird als Indikatorfraktur für eine osteoporotische Stoffwechsellage angesehen, in deren Folge das Risiko, im nächsten Jahrzehnt weitere osteoporotische Frakturen wie eine pertrochantäre Femurfraktur oder Wirbelkörperfrakturen zu erleiden, deutlich steigt.

\section{Abkürzungen}

ALRUD akute longitudinale radioulnare Dissoziation

AO Arbeitsgemeinschaft für Osteosynthesefragen

a.-p. anterioposteriorer Strahlengang

AWMF Arbeitsgemeinschaft der Wissenschaftlichen Medizinischen Fachgesellschaften e. $V$.

CRPS Complex regional Pain Syndrome (komplexes regionales Schmerzsyndrom)

CT Computertomografie

CTS Carpal Tunnel Syndrome (Karpaltunnelsyndrom)

DRUG distales Radioulnargelenk

FCR M. flexor carpi radialis

KD/K-Draht Kirschner-Draht

LT lunotriquetral

MdE Minderung der Erwerbsfähigkeit

MRT Magnetresonanztomografie

SL skapholunär

SLAC Scapholunate advanced Collapse

SNAC Scaphoid non Union advanced Collaps

TFCC Triangular Fibrocartilage Complex (triquetraler fibrokartilaginärer Komplex) 
85\% der Patienten mit distaler Radiusfraktur weisen zum Frakturzeitpunkt eine Kalksalzminderung auf und etwas über die Hälfte eine manifeste Osteoporose.

\section{Diagnostisches Vorgehen}

\section{Symptomatik}

Bei Vorstellung zur Erstbehandlung klagen die Verletzten über Schmerzen und Funktionseinschränkungen im verletzten Handgelenk.

\section{- Fehlstellung}

Typisch ist eine Fourchette- oder Bajonett-Fehlstellung zusammen mit Schwellung und tastbarer Krepitation. Die Bajonettfehlstellung entsteht dadurch, dass das distale Radiusfragment sich speichenwärts und handrückenwärts verschiebt (Abb. 1).

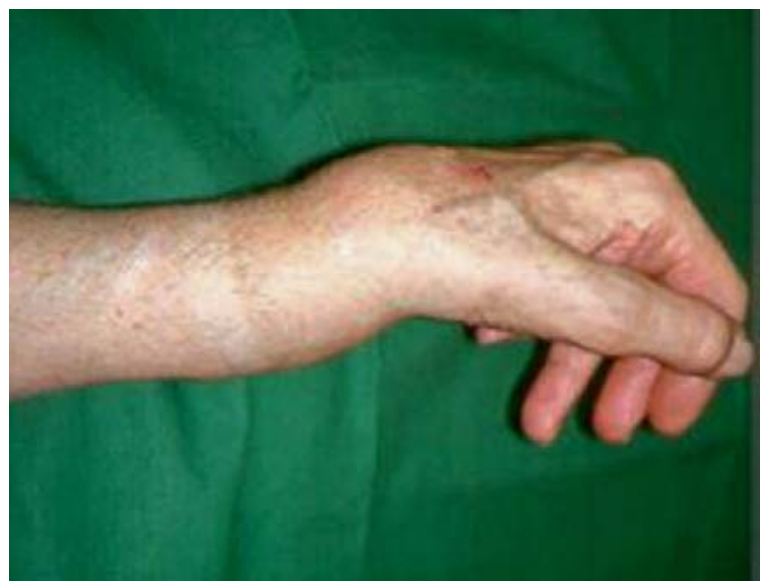

Abb. 1 - Typisches klinisches Bild bei distaler Radiusfraktur (Bajonettfehlstellung).

Checkliste

Mögliche Begleitverletzungen der distalen Radiusfraktur

\begin{tabular}{|l|}
\hline Skaphoidfrakturen \\
Radiusköpfchenfrakturen \\
Verletzungen des triquetralen fibrokartilaginären Komplexes (TFCC) \\
Verletzungen des distalen Radioulnargelenks (DRUG) \\
Beeinträchtigungen des N. medianus \\
zusätzlich vorliegende Verletzungen der Metakarpalia oder Phalangen \\
karpale Instabilität \\
\hline Verletzungen der Ulna bzw. des Radioulnargelenks \\
\hline
\end{tabular}

\section{- Unfallmechanismus}

Zu erfragen ist der Unfallmechanismus. Je nach Stellung der Hand zum Zeitpunkt des Aufpralls entstehen unterschiedliche Frakturtypen. Man unterscheidet zwischen

- Extensionsfrakturen (Colles) und

- Flexionsfrakturen (Smith).

\section{Begleitverletzungen}

Bei der klinischen Untersuchung muss auf Begleitverletzungen geachtet werden, insbesondere auf die in der Checkliste genannten Traumata.

An begleitende unfallbedingte karpale Instabilitäten durch Ruptur der skapholunären Bänder oder des lunotriquetralen Bandapparats muss gedacht werden.

Bei distalen Radiusfrakturen muss immer auch nach einer Fraktur der Ulna gesucht werden.

Dabei ist auf eine Instabilität des distalen Radioulnargelenks zu achten. Eine dislozierte Fraktur des Processus styloideus ulnae kann hinweisend sein auf eine Verletzung des TFCC und damit eine Instabilität des distalen Radioulnargelenks. Die Sonderform der Galeazzi-Verletzung mit Subluxation oder Luxation des Ulnakopfes bei gleichzeitiger Zerreißung der Membrana interossea sollte nicht übersehen werden.

Seltener sind Verletzungen aus dem als ALRUD (akute longitudinale radioulnare Dissoziation) bekannten Formenkreis, die auch als Essex-Lopresti-Verletzung bezeichnet werden. Selten sind gleichzeitige akute Sehnenrupturen oder ein Entrapment einer Sehne zwischen Frakturfragmenten.

Häufiger kommen Sehnenrupturen im Behandlungsverlauf vor. Besonders gefährdet ist die dünnkalibrige Sehne des M. extensor pollicis longus, die durch ihren Verlauf um das Tuberculum Listeri durch scharfe Frakturkanten oder durch das Osteosynthesematerial in Mitleidenschaft gezogen werden kann.

\section{Bildgebende Diagnostik}

\section{- Röntgen}

Besteht der Verdacht auf eine Ulna-minus- oder -plusVariante, kann eine Vergleichsaufnahme der unverletzten Gegenseite hilfreich sein. 


\section{Zielgerichtete Diagnostik}

\section{Konventionelle Röntgenaufnahme}

Mit einer guten konventionellen Röntgenaufnahme des Handgelenks a.-p. und seitlich können die meisten Radiusfrakturen ausreichend beurteilt werden.

\section{- Computertomografie}

Eine CT-Untersuchung empfiehlt sich bei Impressionen oder Trümmerzonen der Gelenkfläche, um den operativen Zugangsweg zu planen. Die CT-Untersuchung ist bei karpalen Begleitverletzungen, insbesondere der Skaphoidfraktur, präoperativ notwendig. Nur in einem Feinschicht-CT lässt sich sicher beurteilen, ob eine Skaphoidfraktur instabil und somit osteosynthetisch versorgungspflichtig ist.

\section{Magnetresonanztomografie}

Die MRT gehört nicht zur Standarddiagnostik bei der distalen Radiusfraktur, kann aber als ergänzende Untersuchung zur Abklärung von ligamentären Begleitverletzungen, insbesondere einer skapholunären Dissoziation, und zur Beuteilung des TFCC hilfreich sein.

\section{- Arthroskopie}

Auch die Arthroskopie ist kein Standarddiagnostikum bei Vorliegen einer distalen Radiusfraktur, gilt aber als sicherste Methode, eine TFCC-Läsion und eine skapholunäre (SL) Bandruptur zu diagnostizieren. Bei zentraler Impression kann die Reposition der Gelenkfläche intraoperativ arthroskopisch beurteilt werden.

\section{Klassifikation}

Von einer Radiusfraktur loco typico spricht man bei einer Fraktur auf Höhe der anatomischen Schwachstelle metaphysär proximal der Strecksehnenfächer. Eine grobe Einteilung unterscheidet nach dem Unfallmechanismus zwischen

- Colles = Extensionsfraktur und

- Smith = Flexionsfraktur.

Eine intraartikuläre Fraktur mit isoliertem Processusstyloideus-radii-Fragment wird Chauffeurfraktur genannt.
Die Einteilung nach Barton unterscheidet zwei intraartikuläre Frakturtypen.

- Bei der Barton-Fraktur ist der dorsale Rand des distalen Radius betroffen, teilweise mit Luxation des Radiokarpalgelenks.

- Bei der umgekehrten Barton-Fraktur ist der palmare Rand des distalen Radius betroffen.

Genauer ist die AO-Klassifikation der distalen Radiusfraktur, für die eine gute Interbeobachterreliabilität und Reproduzierbarkeit nachgewiesen sind, weshalb sie am weitesten Verbreitung gefunden hat.

Die AO-Klassifikation unterscheidet zwischen

- extraartikulären Frakturen

- partiell artikulären Frakturen und

- intraartikulären Frakturen (Tab. 1).

Wir benutzen im folgenden Text diese Einteilung, da sie von den wissenschaftlichen Fachgesellschaften wie der AWMF und in der aktuellen internationalen Literatur häufig verwendet wird und damit Studienergebnisse vergleichbar macht. Es existiert eine Vielzahl unterschiedlicher Klassifikationssysteme für diese häufige Verletzung, z.B. nach Frykman, Melone, Mayo, Pechlaner, um nur einige zu nennen (s. Infobox „Hintergrund“, S. 255). Eine kurze Zusammenfassung der gebräuchlichsten Klassifikationen ist zu finden bei der AWMF unter www.awmf.org/leitlinien.html.

Hilfreich zum besseren Verständnis des Frakturausmaßes erscheint uns das Drei-Säulen-Modell nach Rikli und Reggazoni (1996; Abb. 2). Das Drei-Säulen-Modell ist das

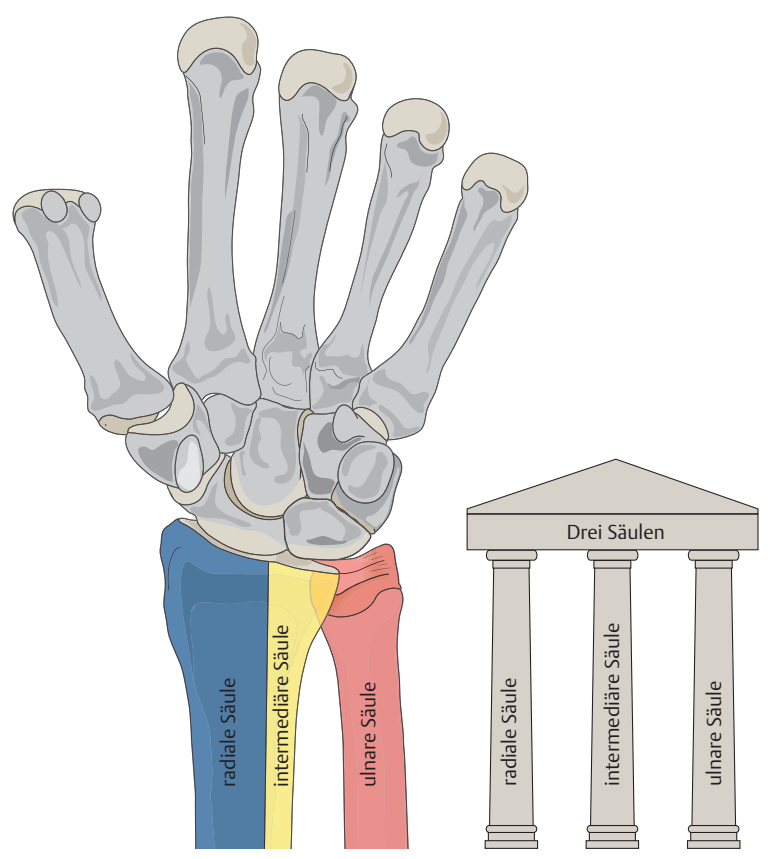

Abb. 2 - DreiSäulen-Modell nach Rikli u. Regazzoni (1996). 


\section{Schultergürtel und obere Extremität}

Tabelle 1

AO-Klassifikation der distalen Radiusfraktur.

Typ

Typ A extraartikuläre Fraktur

A1 Fraktur Ulna

A2 Fraktur Radius einfach

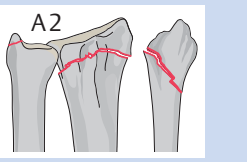

A3 Fraktur Radius mehrfach



Typ B partielle Gelenksbeteiligung

B1 sagittale Radiusfraktur (Chauffeurfraktur/Hutchinson-Fraktur)

B2 Fraktur dorsaler Rand (Barton-Fraktur)

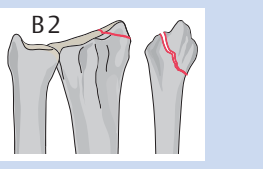

B3 Fraktur palmarer Rand (umgekehrte Barton-Fraktur)

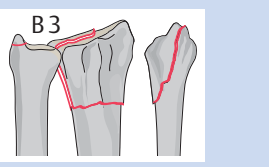

Typ C intraartikuläre Fraktur

C1 artikulär einfach, metaphysär einfach

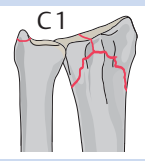

C2 artikulär einfach, metaphysär Mehrfragmentfraktur

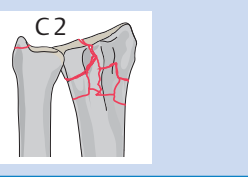

C3 Komplex (Mehrfragmentfraktur)

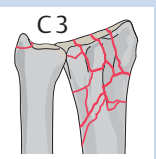

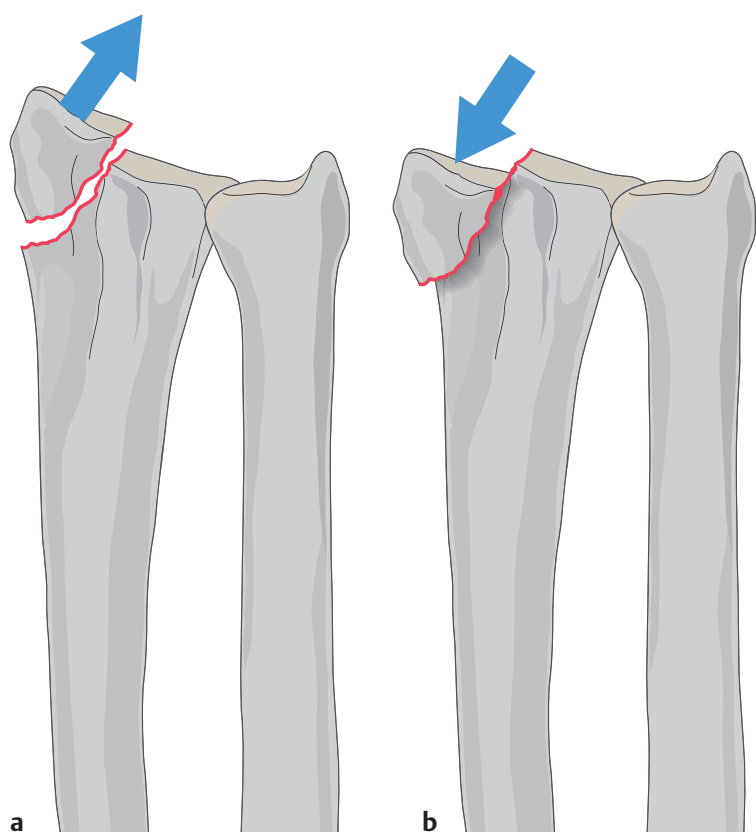

Abb. 3 - Radiusstyloidfrakturen. a Avulsionsverletzung des Radiusstyloids. b Impressionsverletzung des Radiusstyloids.

Resultat biomechanischer Betrachtungen des Unterarms. Es gliedert den distalen Unterarm in 3 Säulen. Die radiale Säule läuft durch den Processus styloideus radii und die Fossa scaphoidea, die zentrale Säule durch die Fossa lunata und die Facies articularis ulnae und die ulnare Säule durch die distale Ulna mit dem ulnokarpalen Komplex.

Die radiale Säule ist für die Stabilität verantwortlich. Sie bietet eine radiale ossäre Abstützung und dient zur Insertion für stabilisierende radiokarpale Bänder. Es gibt 2 Typen von Radiusstyloidfrakturen:

- Die Avulsionsverletzung entspricht einem ossären Abriss der stabilisierenden radiokarpalen Bänder und Abscherung der ossären radialen Abstützung (Abb. 3a).

- Impressionen als Resultat von Kompressionskräften finden wir hingegen nur sehr selten, da die Kraftübertragung nicht über die radiale Säule läuft (Abb. 3b).

Die intermediäre Säule ist Hauptträger der Kraftübertragung von der Hand auf den Unterarm. Bei intraartikulären Frakturen ist das Radiusstyloid meist ein großes, intaktes Fragment und weist keine Impressionen auf. Die Hauptverletzung im radiokarpalen Gelenk liegt im Bereich der intermediären Säule. Hier finden wir eine Aufspaltung in ein palmarulnares und ein dorsoulnares Fragment und häufig auch zentral imprimierte Fragmente durch das Einwirken von Kompressionskräften. 


\section{Weitere Klassifikationssysteme der distalen Radiusfrakturen}

\section{Bewertungsschema nach Lindstrom:}

- Kriterium A (Funktion): Schmerz, Beweglichkeit

- Kriterium B (Röntgen): Verkürzung, Verkippung, Verschiebung

- Kriterium C: Ästhetik, Deviation

\section{Klassifikation nach Frykmann:}

- Typ I: extraartikulär, ohne Ulnafraktur

- Typ II: extraartikulär, mit Ulnafraktur

- Typ III: intraartikulär (radiokarpal), ohne Ulnafraktur

- Typ IV: intraartikulär (radiokarpal), mit Ulnafraktur

- Typ V: intraartikulär (radioulnar), ohne Ulnafraktur

- Typ VI: intraartikulär (radioulnar), mit Ulnafraktur

- Typ VII: intraartikulär (radiokarpal und radioulnar), ohne Ulnafraktur

- Typ VIII: intraartikulär (radiokarpal und ulnokarpal), mit Ulnafraktur

\section{Klassifikation nach Melone:}

- Typ I: stabile Fraktur

- Typ II: instabile Fraktur

- Typ II: A retinierbare Fraktur

- Typ II: B nicht retinierbare Fraktur

- Typ III: instabile Spike-Fraktur

- Typ IV: instabile Mehrfragmentfraktur

- Typ V: Trümmerfraktur

\section{Klassifikation nach Fernandez:}

- 1 - Biegungsfraktur: eine metaphysäre Kortikalis gibt der einwirkenden Zugspannung nach, die Gegenkortikalis weist eine Trümmerzone auf, extraartikuläre Fraktur

- 2 - Abscherfrakturen der Gelenkfläche: Barton; umgekehrte Barton-Frakturen des Processus styloideus, einfache artikuläre Fraktur

- 3 - Kompressionsfrakturen: Fraktur der Oberfläche des Gelenks mit Impaktion der subchondralen und metaphysären Knochen („die-punch“), intraartikuläre Trümmerfrakturen

- 4 - Avulsionsfraktur: Bruch der Bandansätze des ulnaren und radialen Processus styloideus radii; radiokarpale Luxationsfraktur

- 5 - Kombinationen: Kombinationsverletzungen, Hochenergieverletzungen

\section{Mayo-Klassifikation für intraartikuläre Radiusfrakturen:}

- Typ 1: extraartikulär radiokarpale Fraktur, intraartikulär radioulnar

- Typ 2: intraartikulär Fossa scaphoidea

- Typ 3: intraartikulär Fossa lunata \pm sigmoidea

- Typ 4: intraartikulär Fossa scaphoidea, lunata und sigmoidea

\section{Pechlaner-Klassifikation:}

- Typ I-1: dorsale metaphysäre Fraktur

- Typ I-2: dorsale metaphysär-artikuläre Fraktur

- Typ I-3: dorsale Luxationsfraktur

- Typ II-1: zentrale metaphysäre Fraktur

- Typ II-2: zentrale metaphysär-artikuläre Fraktur

- Typ II-2A: zentrale Impressionsfraktur

- Typ II-2B: Fraktur des Processus styloideus radii

- Typ II-2C: ulnarer Randbruch

- Typ II-2D: zentraler Mehrfragmentbruch

- Typ II-3: zentrale Luxationsfraktur

- Typ III-1: palmare metaphysäre Fraktur

- Typ III-2: palmare metaphysär-artikuläre Fraktur

- Typ III-3: palmare Luxationsfraktur

Ergänzende Parameter:

- A: Fraktur unverschoben

- B: Fraktur geschlossen reponierbar und stabil

- C: Fraktur geschlossen reponierbar und/oder instabil

- D: metaphysäre Stauchungszone $<5 \mathrm{~mm}$

- E: metaphysäre Stauchungszone $>5 \mathrm{~mm}$

- F: intraartikuläre Dislokation der Fragmente $<5 \mathrm{~mm}$

- G: intraartikuläre Dislokation der Fragmente $>5 \mathrm{~mm}$

- H: Instabilität des distalen Radioulnargelenks

- I: begleitende karpale Instabilität

Bei der operativen Therapie von intraartikulären Radiusfrakturen gilt die intermediäre Säule deshalb als Schlüssel zum Gelenk.

Die ulnare Säule gewährleistet Kraftübertragung und Stabilität und dient als ulnarer Pfeiler (Abb. 4). 


\section{Schultergürtel und obere Extremität}

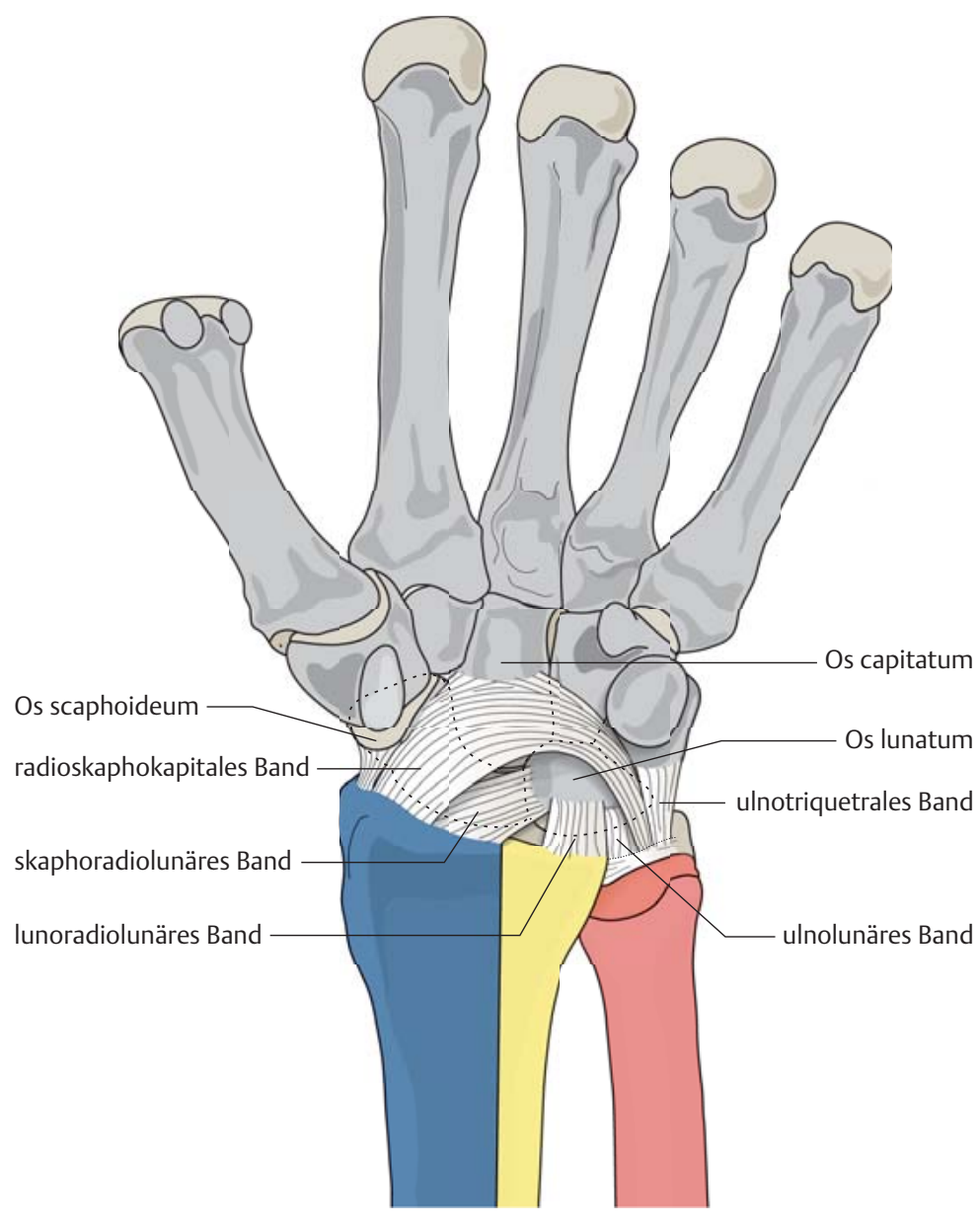

Abb. 4 - Schema der palmaren radiokarpalen Bandverbindungen.

\section{Therapeutisches Vorgehen}

\section{Therapieziele}

Behandlungsziel ist die Frakturheilung mit

- schmerzloser freier Beweglichkeit im Handgelenk,

- uneingeschränkter Unterarmumwendbewegung,

- kraftvollem Faustschluss und

- freier Streckbarkeit der Finger.

\section{Therapieentscheidung}

In der AWMF-Leitlinie Unfallchirurgie zur distalen Radiusfraktur von Mai 2008 wird ein Behandlungsschema vorgeschlagen, das je nach AO-Klassifikation eine unterschiedliche Therapie von geschlossener Reposition mit und ohne Osteosynthese bis zu offener Reposition mit den unterschiedlichsten Osteosyntheseverfahren und Kombination derselben zur Auswahl stellt. Eine wirkliche Therapieempfehlung lässt sich hieraus nicht ableiten.

\section{Indikationen}

Indikationen zur konservativen Therapie der distalen Radiusfraktur

etablierte Indikationen:

- stabile extraartikuläre Fraktur

- nicht/gering dislozierte intraartikuläre Fraktur

- lokale/allgemeine Kontraindikationen gegen eine OP

relative Indikationen:

- primär reponier- und retinierbare Frakturen mit Instabilitätskriterien (Tab. 2)

- Ablehnung der vorgeschlagenen operativen Therapie durch den Patienten.

Auch nach Metaanalyse der aktuellen Literatur kann kein Therapiealgorithmus für die distale Radiusfraktur abgeleitet werden, da keine der vorliegenden Studien eine ausreichende Evidenz für die meisten der benötigten Entscheidungen liefert.

Die distale Radiusfraktur muss als heterogene Verletzung betrachtet werden. Unsere Therapieentscheidung berücksichtigt daher viele Faktoren wie

- den Unfallmechanismus,

- das Frakturmuster,

- die Weichteilverhältnisse,

- Bandinstabilitäten,

- karpale Begleitverletzungen,

- die Knochenqualität,

- das Alter des Patienten sowie dessen Wünsche.

Etablierte Indikationen zur konservativen Therapie sind stabile extraartikuläre Frakturen, nicht oder gering dislozierte intraartikuläre Frakturen und bestehende Kontraindikationen gegen eine Operation (s. Infobox „Indikationen).

Indikationen für eine operative Therapie sind u.a. offene und instabile Frakturen, primär und sekundär dislozierte sowie irreponible Frakturen, daneben Nerven- und Gefäßverletzungen (s. Infobox „Indikationen“, S. 257).

\section{Konservative Therapie}

Bei undislozierten Radiusfrakturen ohne Instabilitätskriterien (Tab. 2) ist die Indikation zum konservativen Vorgehen gegeben. Man legt eine Unterarmgipsschiene in $20^{\circ}$ Dorsalflexion des Handgelenks an, die in Abhängigkeit von der Knochenqualität 4-6 Wochen getragen wird. Die Fingergrundgelenke bleiben frei und können 
Indikationen

\section{Indikationen zur operativen Therapie der distalen Radiusfraktur}

absolute OP-Indikationen:

- offene Fraktur

- instabile Fraktur (hierzu zählen alle Trümmerfrakturen und dislozierten Flexionsfrakturen)

- dislozierte intraartikuläre Fraktur

- irreponible Fraktur

- sekundär dislozierte Fraktur

- begleitende Nervenverletzung

- begleitende Gefäßverletzung

- Durchblutungsstörungen nach Reposition

- komplexe Begleitverletzungen der Handwurzel und des Handgelenks

relative OP-Indikationen:

- beidseitige Frakturen

- Mehretagenverletzung der oberen Extremität

- operationspflichtige lokale Zusatzverletzungen

- Mehrfachverletzungen

- ausdrücklicher Patientenwunsch, etwa aufgrund spezieller beruflicher oder funktioneller Anforderungen

\section{Tabelle 2}

Radiologische Instabilitätskriterien

\begin{tabular}{|l|l|}
\hline Parameter & Wert \\
\hline radialer Höhenverlust & $>2 \mathrm{~mm}$ \\
\hline Änderung der radialen Inklination & $>5^{\circ}$ \\
\hline Verlust der Palmarkippung & $>10^{\circ}$ \\
\hline artikuläre Stufe & $>1-2 \mathrm{~mm}$ \\
\hline Inkongruenz im DRUG & $>1 \mathrm{~mm}$ \\
\hline
\end{tabular}

sofort beübt werden. Diese Gipsschiene wird wenig gepolstert und klassischerweise von dorsal angelegt. $\mathrm{Zu}$ achten ist auf eine gute Anmodellierung der Schiene und eine engmaschige Kontrolle. An die Ruhigstellung schließt sich eine physiotherapeutische Beübung an.

\section{Notfalltherapie dislozierter Frakturen}

Dislozierte Frakturen werden zunächst am Unfalltag im Aushang in Bruchspaltanästhesie reponiert und dann im Unterarmspaltgips retiniert. Brüske wiederholte Repositionsmanöver ohne ausreichende Schmerztherapie sind dabei zu vermeiden. Bei ungenügender Reposition ist eine Fixateuranlage zu erwägen.

In Abhängigkeit vom Frakturtyp kann eine interne Osteosynthese im Verlauf notwendig werden. Eine OP ist dann indiziert, wenn eine ausreichende Reposition nicht erzielt und eine Retention mithilfe geschlossener Maßnahmen nicht gehalten werden kann. Dies ist bei instabilen Frakturen fast immer der Fall. Eine Flexionsfraktur gilt immer als instabil. Die radiologischen Instabilitätskriterien sind in Tab. 2 aufgelistet.

Ziele einer operativen Frakturbehandlung sind die anatomische Rekonstruktion und die Ermöglichung einer frühfunktionellen Nachbehandlung, um zügig Beweglichkeit und Kraft wiederzuerlangen. Angestrebt wird eine Wiederherstellung der physiologischen Winkel am distalen Radius, also einer radioulnaren Inklination von ca. $24^{\circ}$ und einer Palmarinklination von ca. $12^{\circ}$. Gelenkstufen sollen durch den operativen Eingriff behoben werden.

Offene Frakturen werden zusammen mit der Weichteilverletzung notfallmäßig versorgt, um die Entwicklung einer Wundinfektion und Osteomyelitis zu verhindern. Eine begleitende Medianusläsion zieht eine unaufgeschobene Frakturversorgung mit Dekompression des Nervs nach sich.

\section{Kirschner-Draht-Osteosynthese}

Diese Operationsmethode eignet sich zur Behandlung von A2-Frakturen. Die Kirschner-Drähte (K-Drähte, KD) werden perkutan oder über eine Stichinzision eingebracht. Insertionspunkt ist der Processus styloideus radii. Die Drähte verlaufen in einem Winkel von $30-45^{\circ}$ zur Radiusachse (Abb. 5). Zu vermeiden ist ein Kreuzen der Drähte auf Frakturniveau. Die Drähte werden gekürzt und nicht umgebogen, wenn man sie unter die Haut versenken will.

Bei der Einbringung der Drähte perkutan und vor allem bei ihrer Entfernung ist der R. superficialis des N. radialis verletzungsgefährdet.

Versenkt man die Drähte nicht unter die Haut, ist die Metallentfernung einfacher, der R. superficialis hierbei weniger gefährdet. 


\section{Schultergürtel und obere Extremität}

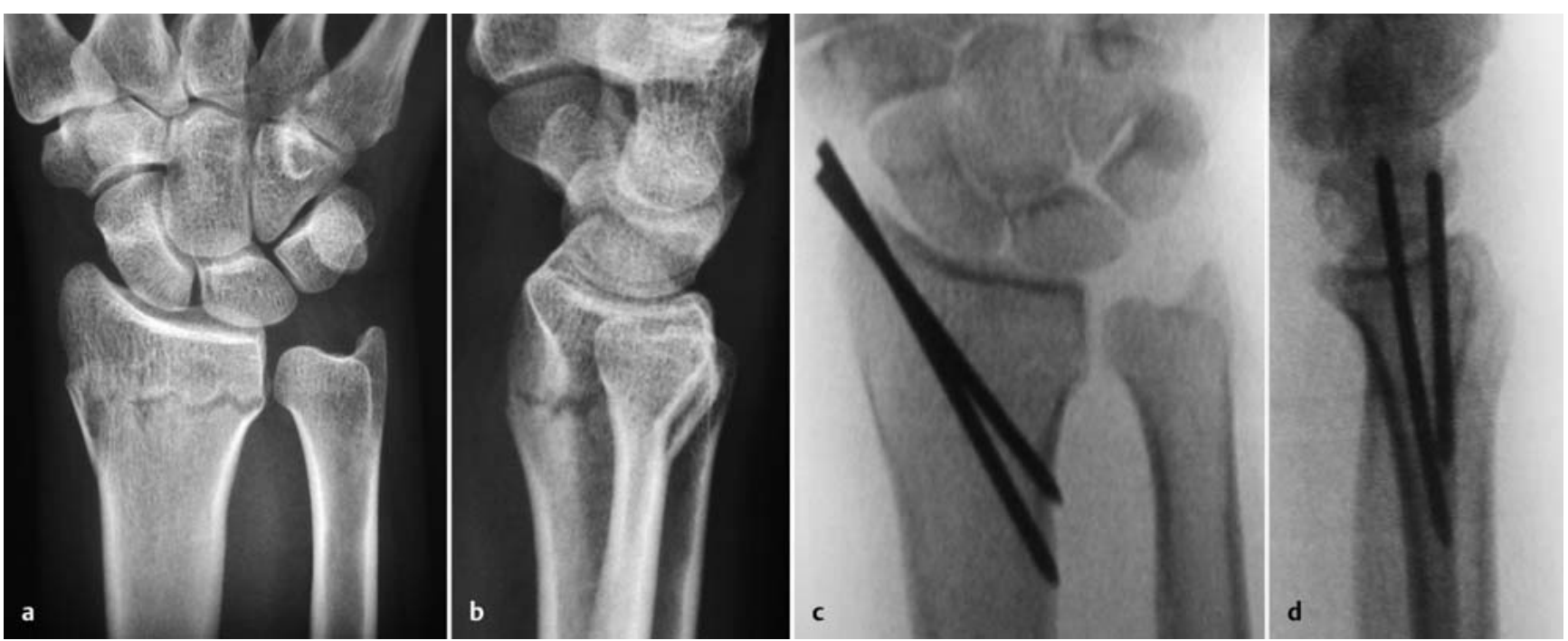

Abb. 5 - A2-Fraktur nach AO; versorgt mit Kirschner-Draht-Osteosynthese. a u. b Präoperative Röntgenaufnahmen a.-p. und seitlich. c u. d Postoperative Röntgenbilder a.-p. und seitlich.

Überragen die Drähte das Hautniveau, muss eine engmaschige Kontrolle und Pflege der Eintrittsstelle sichergestellt sein, um Infektionen zu vermeiden.

Postoperativ ist eine 4- bis 6-wöchige Gipsruhigstellung notwendig. Anschließend erfolgen die Metallentfernung in Lokalanästhesie und eine physiotherapeutische Übungsbehandlung.

\section{- Besonderheiten}

- Vor allem bei osteoporotischem Knochen können eine sekundäre Drahtdislokation und ein Repositionsverlust eintreten. Dann muss eine Revision mit Umstieg auf ein anderes Verfahren erwogen werden.

- Bei dorsalen Trümmerzonen kann das Einbringen intrafokaler Karpandji-Drähte die Reposition ermöglichen. Die intrafokale K-Drahtung ist technisch anspruchsvoll.

- Bei C-Frakturen müssen Gelenkfragmente durch zusätzliche parallel zur Gelenkfläche verlaufende Drähte gefasst werden. Eine Übungs- oder gar Belastungsstabilität lässt sich hierdurch nicht erreichen.

\section{Schraubenosteosynthese}

Eine Schraubenosteosynthese kann bei isolierten Frakturen des radialen Pfeilers eingesetzt werden, insbesondere bei sogenannten Chauffeurfrakturen (Abb. 6). Die Kleinfragmentschrauben werden über eine $2-3 \mathrm{~cm}$ lange Inzision über dem Processus styloideus unter Durchleuchtungskontrolle eingebracht. Gefährdete Strukturen sind der R. superficialis des N. radialis und die Sehnen des M. abductor pollicis longus und M. extensor pollicis brevis.

Die Nachbehandlung kann frühfunktionell belastungsfrei erfolgen. Eine Implantatentfernung sollte nach Konsolidierung der Fraktur empfohlen werden.

\section{Fixateur externe}

Komplexere Frakturen können mit einem gelenküberbrückenden Fixateur externe versorgt werden. Dieses Verfahren eignet sich gut zur Primärstabilisierung, der nach Weichteilkonsolidierung interne Osteosynthesen folgen können. Auch die Ausbehandlung im Fixateur ist möglich.

Die Reposition erfolgt durch Ligamentotaxis. Vorteil ist, dass es sich um eine minimalinvasive Methode handelt, die kaum ein zusätzliches Weichteiltrauma setzt. Auch bei komplexen Frakturen können gute Repositionsergebnisse erzielt werden. Das Konstrukt kann durch zusätzliche perkutane Kirschner-Drähte verstärkt werden (Abb. 7 u. Abb. 8). Biomechanisch soll dadurch die Stabilität der Montage vergleichbar mit einer dorsalen 3,5-mmT-Platte werden. Der Einrichtung von Trümmerzonen der Gelenkfläche sind jedoch methodische Grenzen gesetzt. Die Einrichtung zentraler Fragmente gelingt nicht. Auch ein sekundärer Korrekturverlust ist nicht selten.

Eine frühfunktionelle Nachbehandlung ist verfahrensbedingt nicht möglich. 




Abb. 6 - B1.1-Fraktur nach AO; versorgt mit Schraubenosteosynthese. a u. b Präoperative Röntgenaufnahmen a.-p. und seitlich. c u. d Postoperative Röntgenbilder a.-p. und seitlich. e u. f Röntgenbilder nach Implantatentfernung.

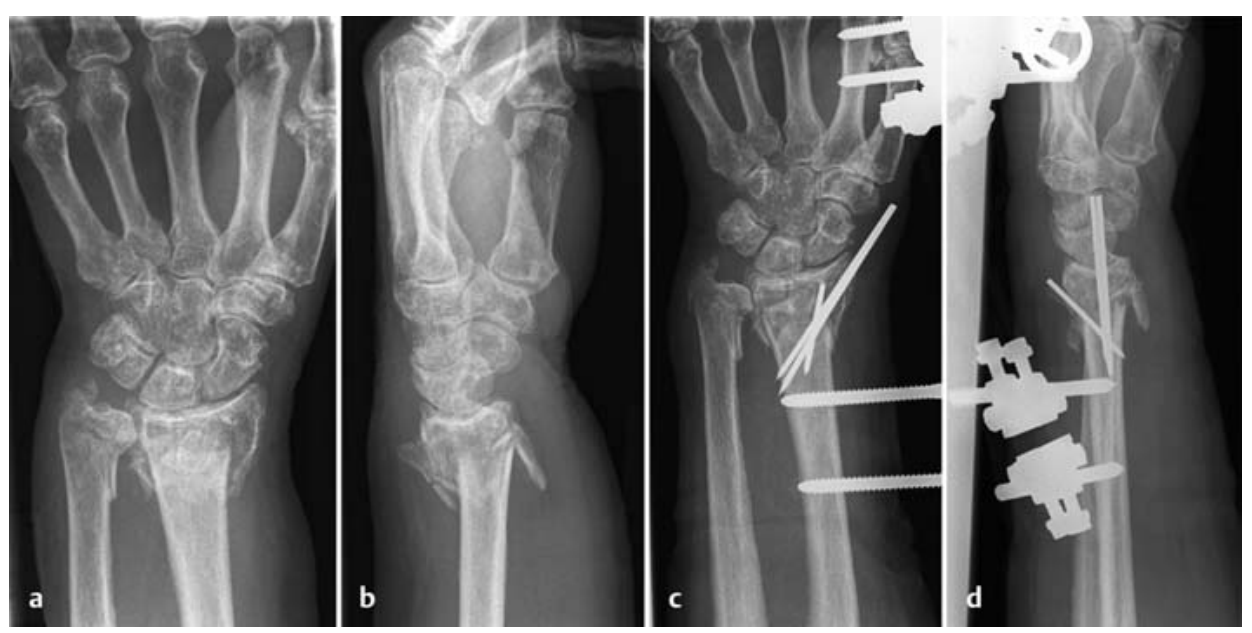

Abb. 7 - C3-Fraktur nach AO; versorgt mit Fixateur externe und Kirschner-

Drähte.

a u. b Präoperative Röntgenaufnahmen a.-p. und seitlich.

c u. d Postoperative Röntgenbilder a.-p. und seitlich.


Abb. 8 a C3-Fraktur nach AO; versorgt mit Fixateur externe und Kirschner-Drähten. a u. b Präoperative Röntgenaufnahmen a.-p. und seitlich. c u. d Postoperative Röntgenbilder a.-p. und seitlich. e u. f Ausheilungsbilder a.-p. und seitlich. 


\section{Schultergürtel und obere Extremität}

- Besonderheiten

- Eine Kombination mit palmar abstützenden Platten kann bei Trümmerfrakturen (C3) sinnvoll sein.

- Alternativ kann ein nicht gelenküberbrückender Fixateur externe verwendet werden. Die Operationsmethode ist ungleich aufwendiger. Zunächst wird ein gelenküberbrückender Fixateur externe montiert. Die Ligamentotaxis kann optimal ausgenutzt werden. durch Distraktion und Flexion (Cotton-Lodder-Stellung). Dann werden die distalen Fragmente mit mindestens 4 Kirschner-Drähten fixiert, die über Ausleger an der proximalen Fixateurmontage befestigt werden Anschließend wird die gelenküberbrückende Repositionshilfe entfernt (Abb. 9). Die Beübung des Handgelenks ist vom Tag der Operation an möglich (Fallbeispiel Abb. 10). In Studien zeigten sich als Vorteile dieses Verfahrens gegenüber dem gelenküberbrückenden Fixateur externe die frühfunktionelle Nachbehandlungsmöglichkeit und die etwas bessere Rekonstruierbarkeit der radialen Länge ohne das Risiko der Überdistraktion.

\section{Palmare winkelstabile Plattenosteosynthese}

Dieses Verfahren ermöglicht eine anatomische Rekonstruktion und bei fehlenden Begleitverletzungen eine frühfunktionelle Nachbehandlung. Durch die Winkelstabilität wird ein weniger fester Halt im Knochen benötigt als bei herkömmlichen nicht winkelstabilen Systemen, sodass diese Methode auch bei osteoporotischer Knochenstruktur, zur Überbrückung von Trümmerzonen und zum Teil auch bei Extensionsfrakturen vom palmaren Zugang her anwendbar ist. Die Platte dient zudem bei Trümmerzonen als „internal Buttress“.

Eine palmare Plattenosteosynthese ist aufgrund des Frakturverlaufs ideal zur Versorgung von Flexionsfrakturen geeignet. Für die Abstützung der meisten Flexionsfrakturen eignen sich konventionelle und winkelstabile Implantate. In der Literatur werden die radiologischen Ergebnisse als signifikant besser angegeben als nach konservativer Therapie.
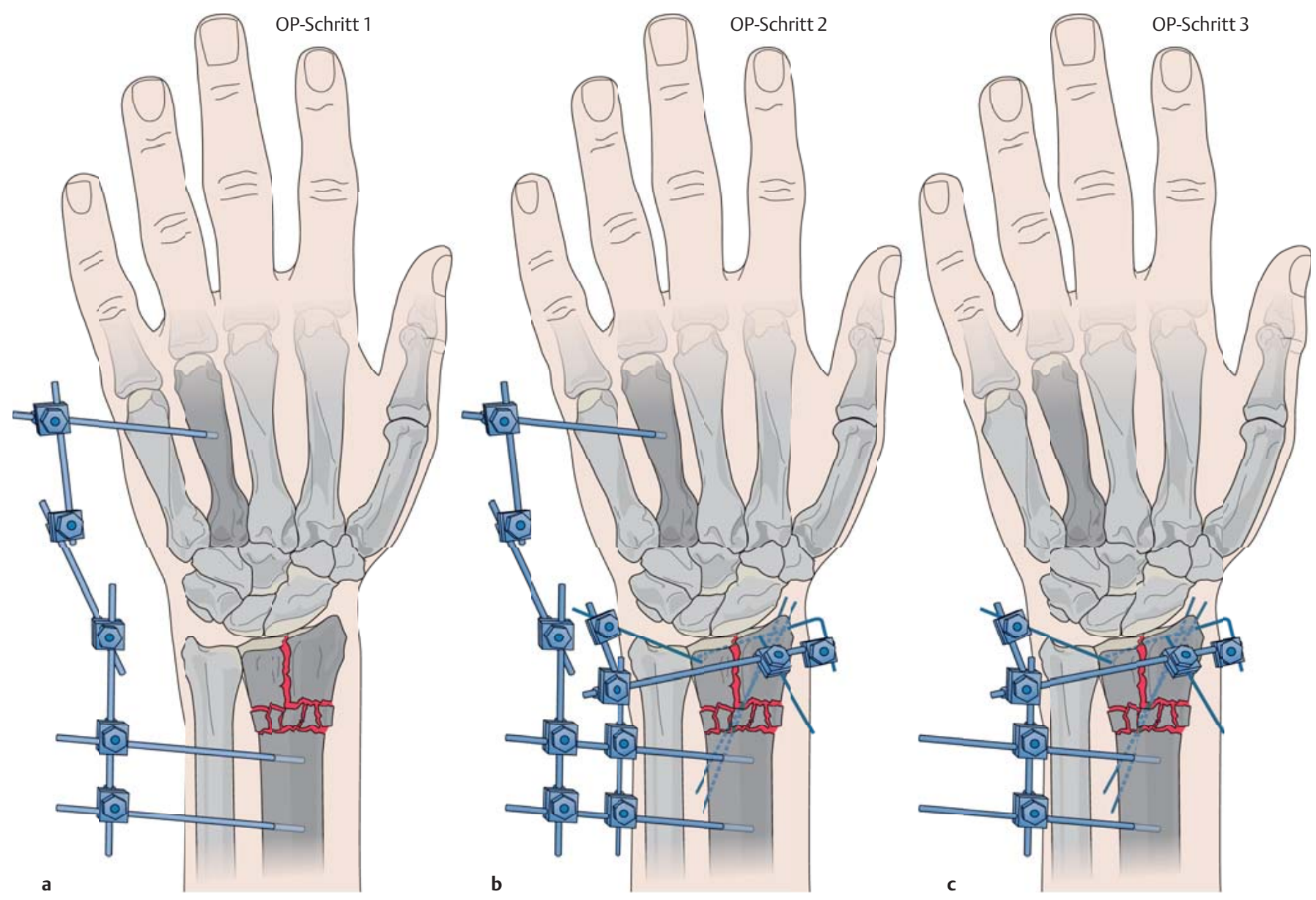

Abb. 9 - Nicht gelenküberbrückender Fixateur externe - Operationsprinzip. a OP-Schritt 1. b OP-Schritt 2. c OP-Schritt 3. 


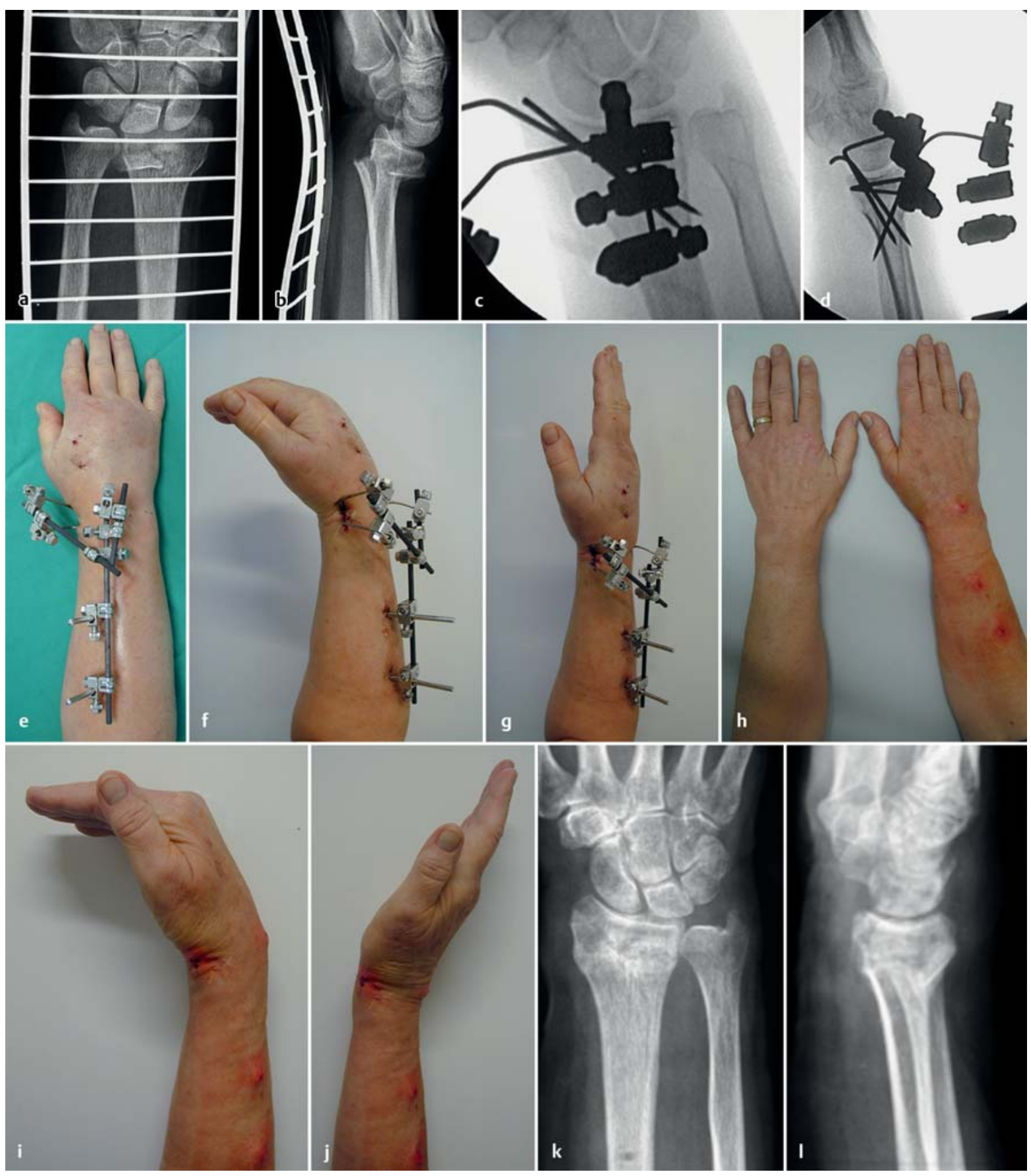

Abb. 10 - AO C2.1-Fraktur nach AO (71 Jahre, weiblich); versorgt mit nicht gelenküberbrückendem Fixateur externe. a u. b Präoperative Röntgenaufnahmen a.-p. und seitlich. c u. d Intraoperative Bildwandleraufnahmen. e-g Funktionsaufnahmen 1. postoperativer Tag. h-j Funktionsaufnahmen nach Implantatentfernung 6 Wochen postoperativ. $\mathbf{k}$ u. I Röntgenbilder nach Konsolidierung und Implantatentfernung a.-p. und seitlich.

Der operative Zugangsweg verläuft radiopalmar entlang der Flexor-carpi-radialis-Sehne, deren Sehnenfach eröffnet wird und die anschließend nach ulnar gehalten wird. Der darunter liegende M. pronator quadratus wird vom Radius abgeschoben (Abb. 11). Der palmare Zugang birgt gegenüber dem dorsalen die Vorteile einer besseren Weichteildeckung des Implantats, eine geringere Gefahr von Sehnenirritationen und bei palmar meist einfach frakturierter Kortikalis eine gute Kontrolle der Frakturreposition (Fallbeispiel s.Abb. 12). 


\section{Schultergürtel und obere Extremität}

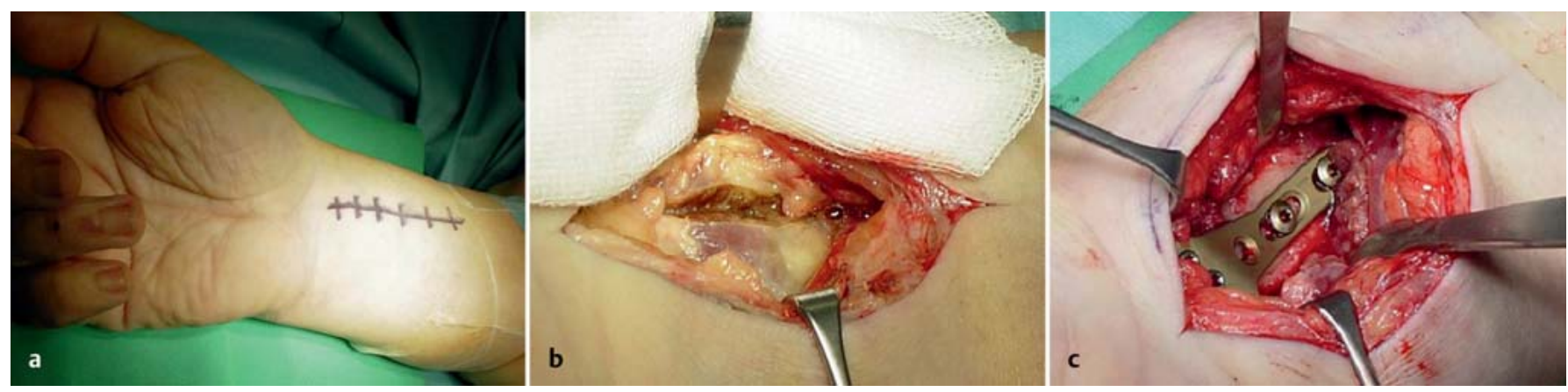

Abb. 11 - Palmarer Zugang entlang der FCR-Sehne. a Angezeichnete Inzision. b Palmarer Zugang, Faszie des M. pronator quadratus eröffnet. c Winkelstabile palmare Platte in situ.
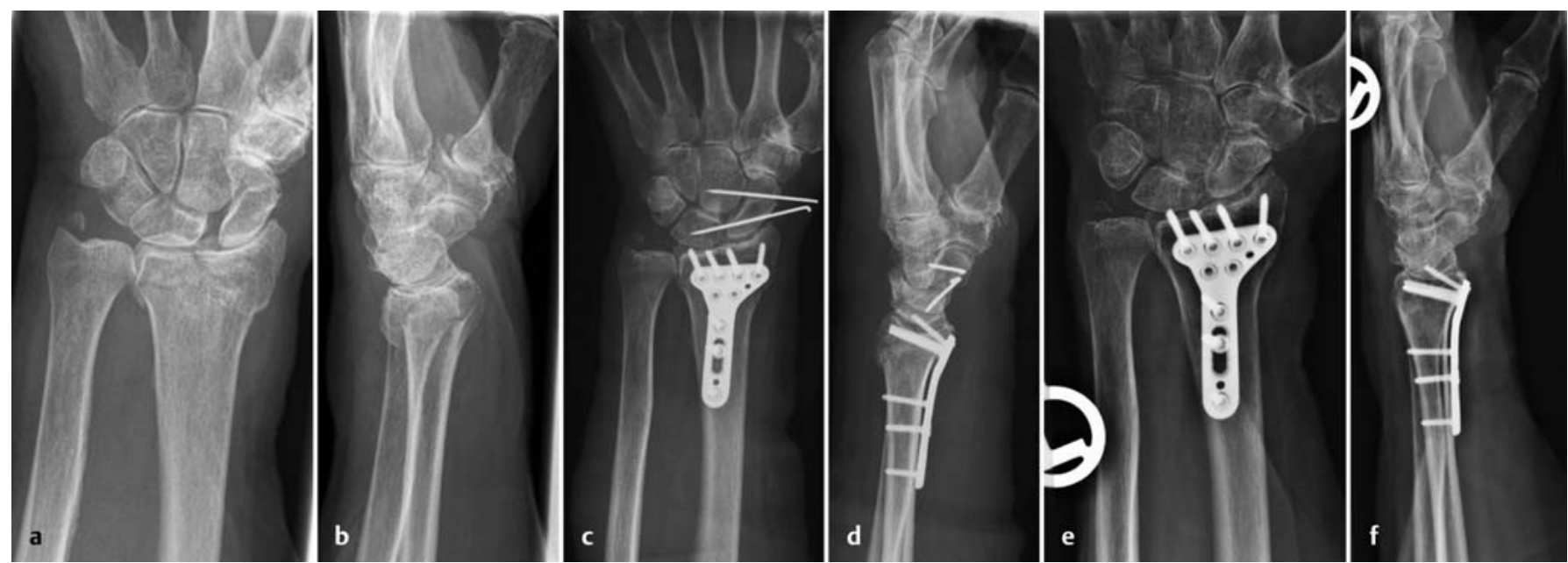

Abb. 12 - C1.2-Fraktur nach AO, begleitende dynamische skapholunäre Dissoziation (81 Jahre, weiblich); versorgt mit palmarer Platte. a u. b Präoperative Röntgenaufnahmen a.-p. und seitlich. c u. d Postoperative Röntgenbilder a.-p. und seitlich. e u. f Röntgenbilder 2⿺辶⿸厃𠄌 Jahre postoperativ. a.-p. und seitlich.
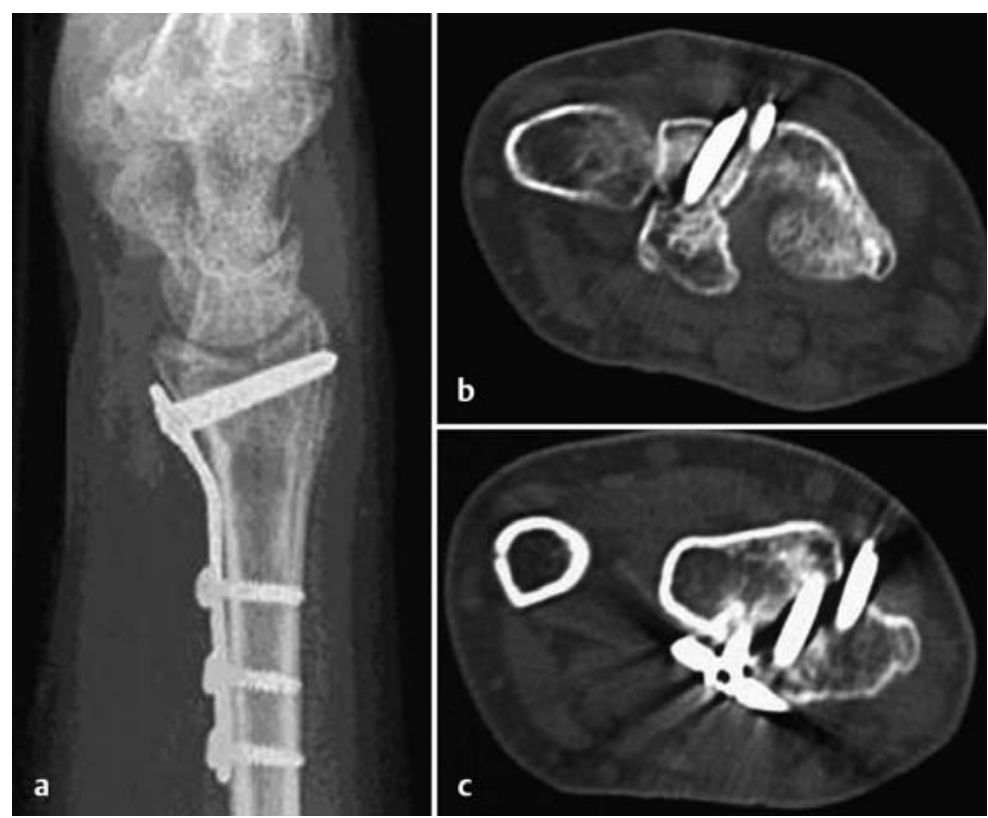

Abb. 13 - Palmare Platte, dorsal die Kortikalis überragende Schrauben. a Konventionelles Röntgenbild. b u. c CT.
- Bei der Plattenplatzierung ist darauf zu achten, die modernen winkelstabilen Platten nicht zu weit distal anzubringen.

Polyaxiale Implantate gewähren einen größeren Spielraum in der Platzierung als monoaxiale. Eine zu weit distale Positionierung an der palmaren Radiuskante (der sogenannten Watershed Line) gilt als fehlerhaft und kann zu Reizungen der Beugesehnen führen. Die Platte sollte mittig auf dem Radius proximal dieser Linie positioniert sein.

Zudem muss sich der Operateur vor Augen halten, das die dorsale Radiusfläche nicht plan konfiguriert ist, sodass auch im Röntgenbild korrekt lang wirkende Schrauben dorsal die Kortikalis überragen und Sehnenirritationen verursachen können (Abb. 13).

- Es wird empfohlen, die distalen Schrauben $2 \mathrm{~mm}$ kürzer zu wählen, als es der intraoperativen Messung entspricht. 


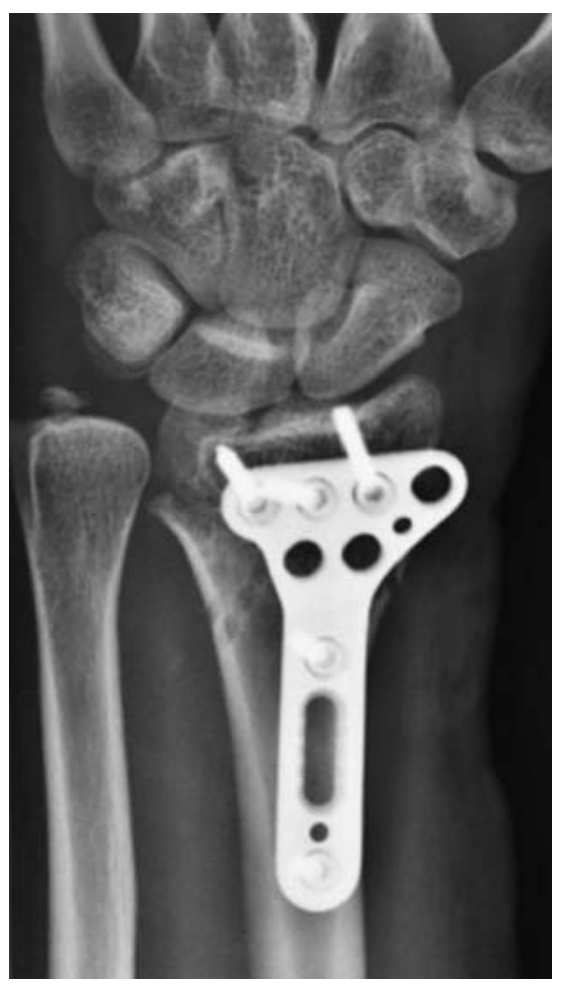

Abb. 14 - Überragen der palmaren Platte radial am distalen Radius.

Auch ein Überragen der Platte radial-distal kann vor allem bei schlanken Patienten als störend empfunden werden und macht eine frühzeitige Implantatentfernung notwendig (Abb. 14).

\section{Intramedulläre Osteosynthese}

Bei extraartikulären A2- und A3- sowie wenig dislozierten C1- und C2-Frakturen ist eine Marknagelosteosynthese mit speziell für den distalen Radius entwickelten Implantaten möglich. Eigene Erfahrung haben wir mit dem Marknagel Targon DR.

Die Reposition der Fraktur erfolgt manuell durch Ligamentotaxis. Es können zusätzlich temporär KirschnerDrähte eingebracht werden. Die Hautinzision ist $4 \mathrm{~cm}$ lang und liegt über der Spitze des Processus styloideus radii, über dessen Zentrum ein Führungsdraht eingebracht wird. Hierüber wird die Nageleintrittsstelle mit kanüliertem Bohren vorbereitet. Das Nagellager wird mit Raffeln stufenweise aufgearbeitet und anschließend der Marknagel eingebracht. Über ein Zielgerät werden Drähte eingebracht, die nach Längenmessung durch Schrauben ausgetauscht werden. Die distalen Schrauben werden so platziert, dass sie die Gelenkfläche unterstützen. Proximal werden in den distalen Schaft des Radius analog zu anderen Marknägeln ebenfalls über das Zielinstrument zwei Verriegelungsschrauben eingebracht, um eine Rotationsstabilität zu gewährleisten.

- Bei der Einbringung der distalen, die Gelenkfläche unterstützenden Schrauben muss darauf geachtet werden, die Schrauben nicht zu lang zu wählen, damit das distale Radioulnargelenk nicht beeinträchtigt wird.

Die Nachbehandlung erfolgt gipsfrei frühfunktionell auch bei osteoporotischem Knochen.

Die radiologischen und funktionellen Ergebnisse stellen sich nach aktueller Studienlage analog zu den Ergebnissen mit palmarer Plattenosteosynthese dar. Die Palmarinklination kann bei höhergradig instabilen Frakturen etwas schlechter wiederhergestellt werden. Die Rate der weichteilbedingten Komplikationen, insbesondere der sekundären Sehnenrupturen, scheint geringer auszufallen (Abb. 15).

\section{Dorsale winkelstabile Plattenosteosynthese}

Nicht jede Fraktur lässt sich über einen palmaren Zugang korrekt reponieren. Bei dorsal betonter Pathologie ist ein dorsaler Zugang daher günstiger. Nachdem in der Vergangenheit dorsale Plattenosteosynthesen häufig mit Strecksehnenkomplikationen vergesellschaftet waren, wurde eine optimierte dorsale Plattenlage (Abb. 16) entwickelt und spezielle hierfür geeignete winkelstabile Implantate, die eine sichere Retention der Frakturfragmente möglich machen unter bestmöglichem Schutz der Strecksehnen.

Der dorsale Zugang liegt etwas radial der Handgelenksmitte. Das Retinaculum extensorum wird treppenförmig gespalten, die Strecksehnen werden in ihren Strecksehnenfächern einschließlich des Periosts vom distalen Radius abpräpariert und beiseite gehalten. Es kann notwendig sein, das Tuberculum Listeri zur besseren Plattenplatzierung mit einem Luer zu glätten. Bei Trümmerfrakturen wird von vielen Autoren eine gleichzeitige selektive Denervierung des N. interosseus posterior empfohlen, der problemlos nach Luxation der Strecksehnen auf Höhe des DRUG identifiziert werden kann.

Über den dorsalen Zugang lässt sich die radiokarpale Gelenkfläche gut visualisieren, wenn man die Handgelenkskapsel türflügelartig inzidiert und zur Seite präpariert, sodass sie hinterher wieder verschlossen werden kann. 


\section{Schultergürtel und obere Extremität}
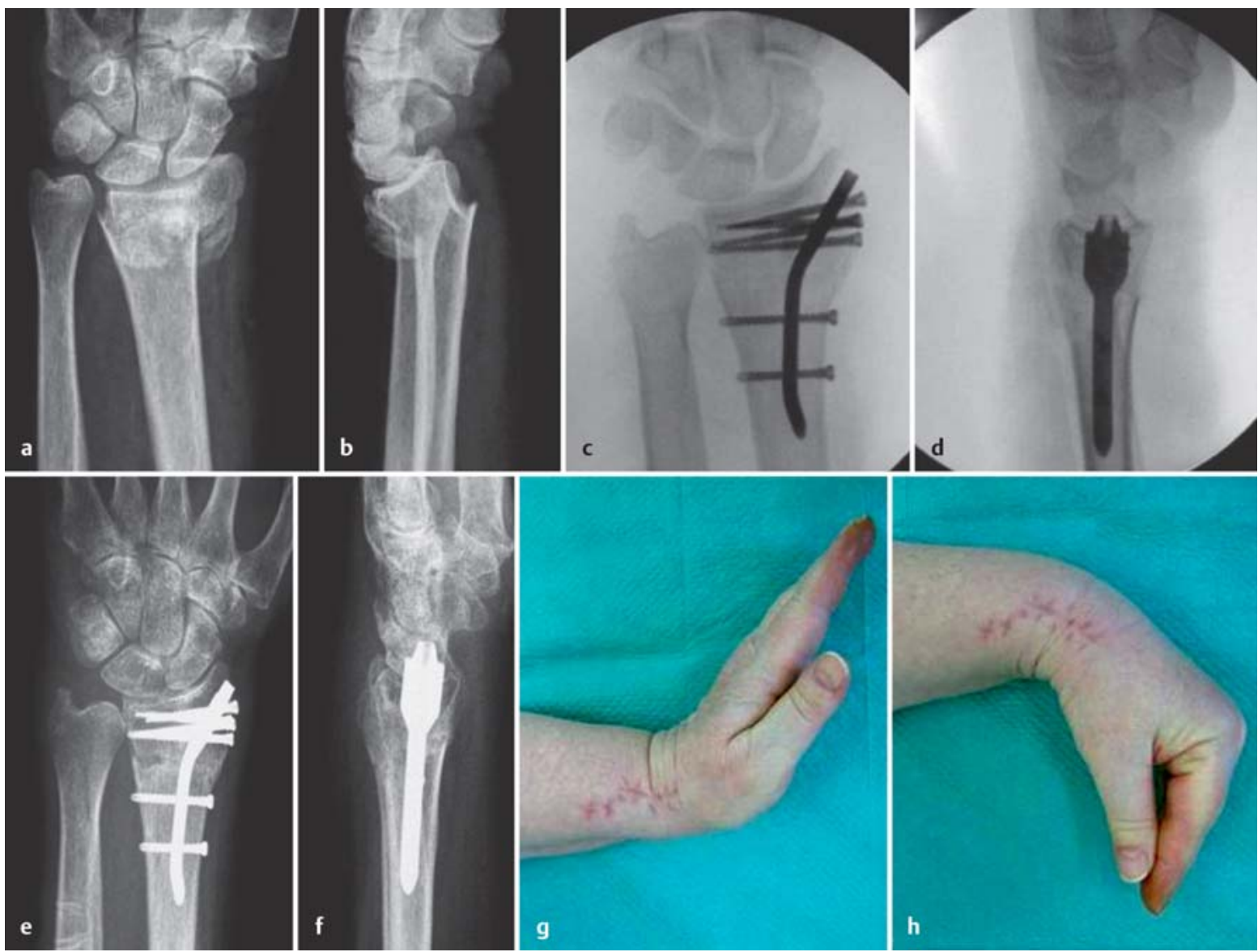

Abb. 15 - A3.1-Fraktur nach AO (82 Jahre, weiblich); versorgt mit Targon DR. a u. b Präoperative Röntgenbilder. c u. $\mathbf{d}$ Intraoperative Bildwandler-Aufnahmen. e u.f Röntgenaufnahmen 12 Wochen postoperativ. $\mathbf{g}$ u. h Funktionsaufnahmen 6 Wochen postoperativ.

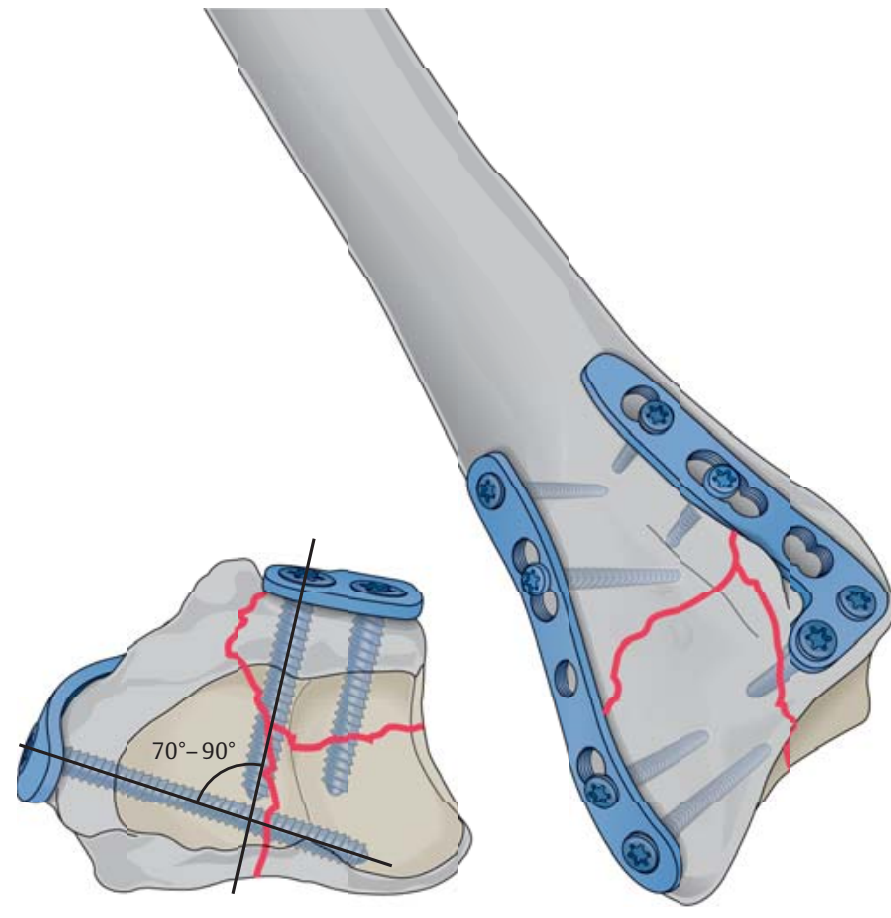

Abb. 16 - Optimale Lage der dorsalen Doppelplattenosteosynthese.
Die gelenktragenden Frakturfragmente werden anatomisch genau eingepasst, präliminär mit Kirschner-Drähten fixiert und dann mit einer winkelstabilen Doppelplattenosteosynthese versorgt, wobei eine L- oder hockeyschlägerförmige Platte dorsal angebracht wird und sich die zweite Platte anatomisch vorgeformt an die radiale Kortikalis - also um $90^{\circ}$ versetzt - anschmiegt. Hierzu wird das 1. und 2. Strecksehnenfach möglichst nicht eröffnet, sondern mit dem Periost vom Radius abgeschoben. Die optimale Plattenlage ist in Abb. 16 skizziert. Nach Verschluss der Handgelenkskapsel wird das Retinaculum unter den Strecksehnen über der Platte vernäht, um ein Aufreiben der Sehnen auf den Platten zu vermeiden.

Die 2,4 mm starken Platten tragen wenig auf. Trotzdem wird eine Implantatentfernung nach Konsolidierung empfohlen. Ein Fallbeispiel ist in Abb. 17 dargestellt. 


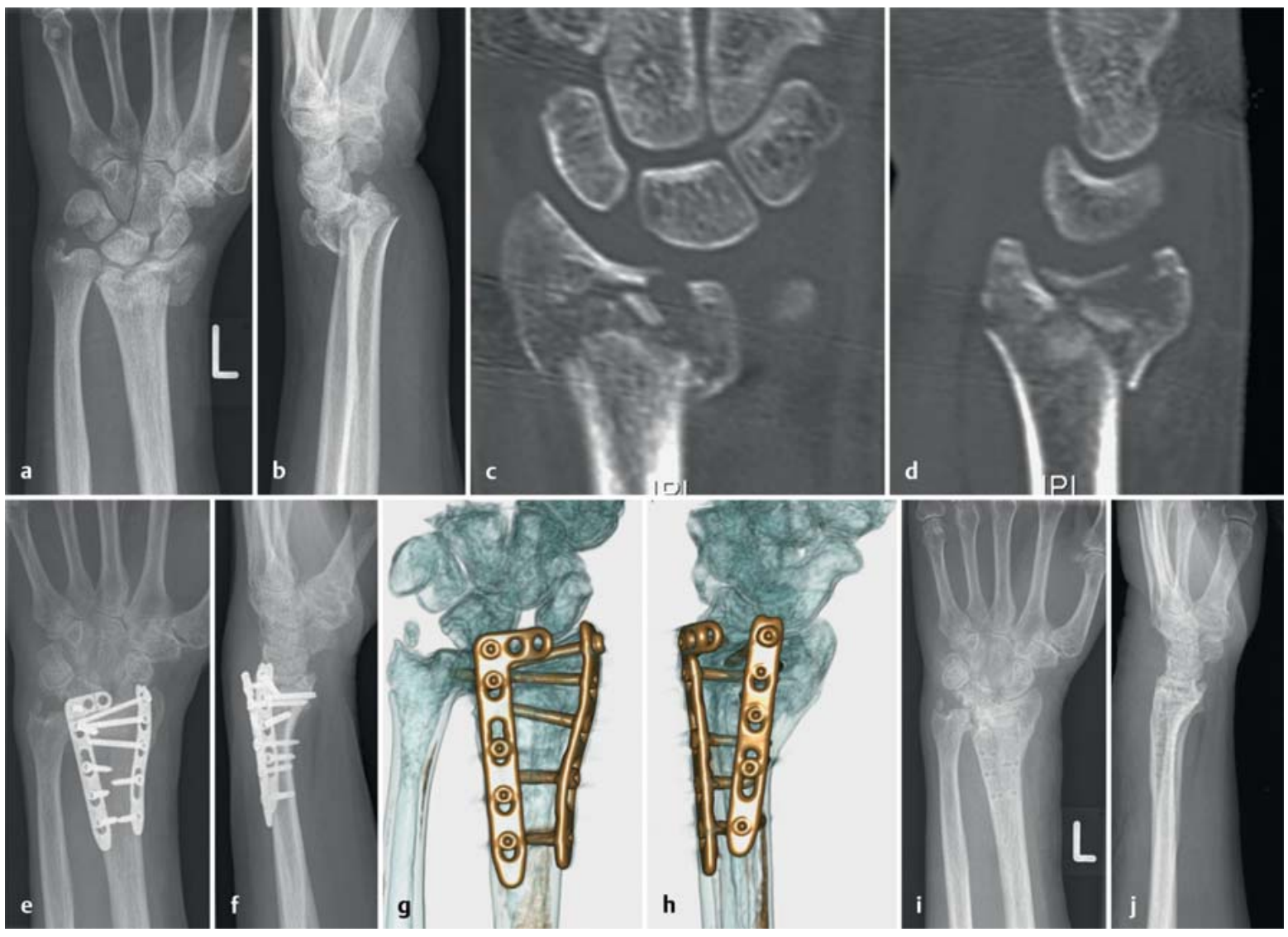

Abb. 17 - C3.1-Fraktur nach AO (62 Jahre, weiblich); versorgt mit dorsaler Plattenosteosynthese. a u. b Präoperatives Röntgenbild in 2 Ebenen. c u. d CT nach Fixateur-Anlage. e u. f Postoperatives Röntgenbild in 2 Ebenen. $\mathbf{g}$ u. h 3-D-Rekonstruktion postoperativ. i u. j Röntgenaufnahmen nach Konsolidierung und Implantatentfernung.

\section{Komplexe Frakturen}

Bei komplexen Frakturen wird oft ein mehrzeitiges Vorgehen notwendig. In der Notfallsituation wird vorzugsweise ein gelenküberbrückender Fixateur externe montiert. Während der Weichteilkonsolidierungsphase kann die weiterführende Diagnostik mittels CT erfolgen und in einem zweiten Schritt die der Fraktur angepasste interne Osteosynthese.

Intraoperativ wird entschieden, ob der Fixateur trotz interner Osteosynthese noch zeitweilig belassen wird oder gegen eine Gips- oder Castschiene ausgetauscht werden kann. Dies ist abhängig von der erreichbaren Primärstabilität der Osteosynthese, insbesondere jedoch von den karpalen Begleitverletzungen (Abb. 18).

\section{Implantatentfernung}

Plattenosteosynthese. Eine Implantatentfernung nach Konsolidierung sollte bei palmarer Plattenlage nicht generell empfohlen werden. Bei dorsaler Plattenosteosynthese sollte eine Implantatentfernung jedoch auch bei älteren Patienten empfohlen werden, da die anatomisch enge Beziehung der Platten zu den Strecksehnen dauerhaft eine Schädigung der Strecksehnen zur Folge haben kann. Die dorsale Plattenosteosynthese geht zugangsbedingt mit einer höheren Rate an Strecksehnenverwachsungen einher.

\section{Fixateur externe und Kirschner-Draht-Osteosynthese.}

Die notwendige Implantatentfernung ist bei Fixateurund Kirschner-Draht-Osteosynthese immer mit aufzuklären.

Schraubenosteosynthese. Auch bei Schraubenosteosynthese isolierter Radiusstyloidfrakturen sollte eine Implantatentfernung geplant werden. Hier sind Verletzun- 


\section{Schultergürtel und obere Extremität}
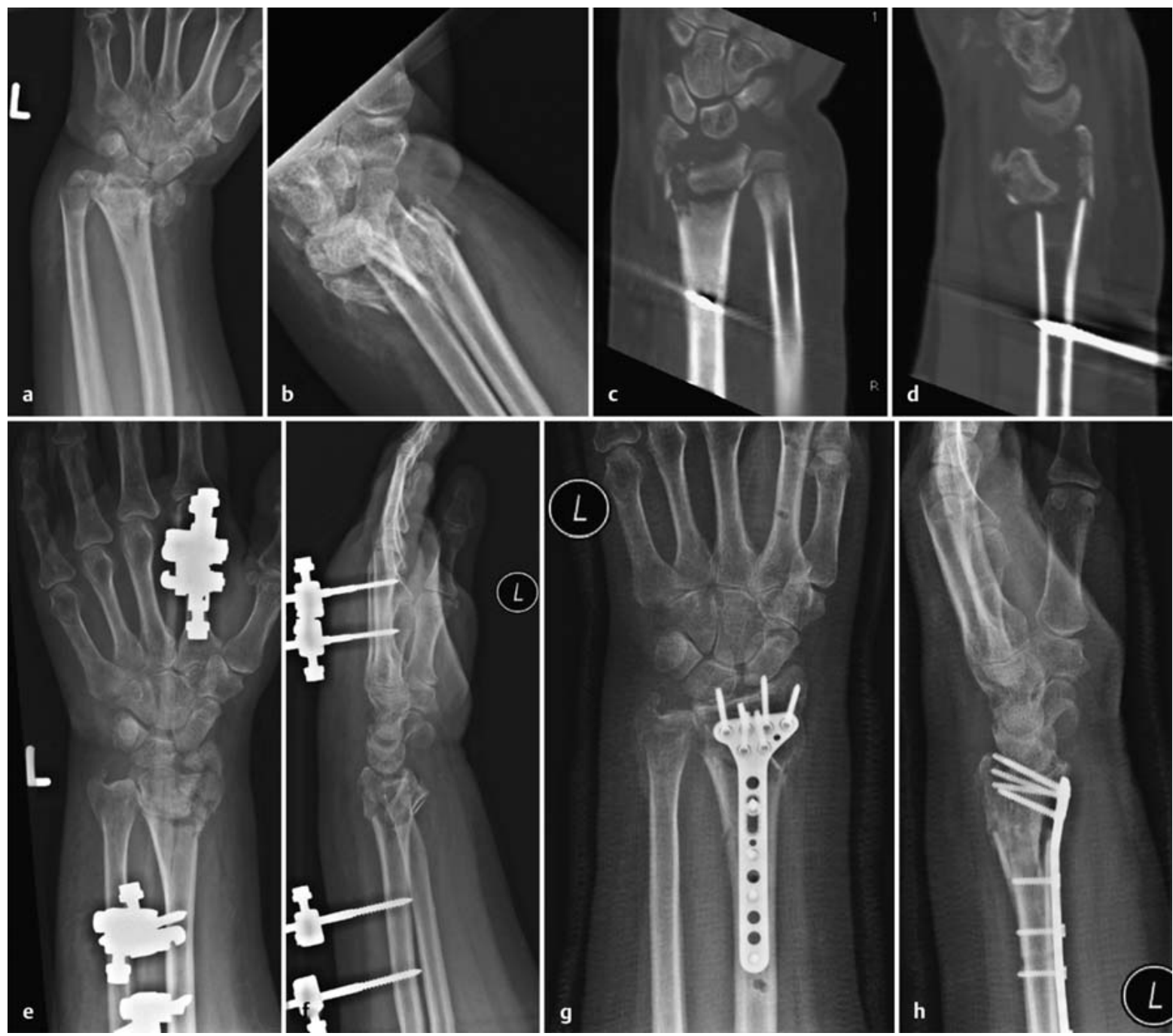

Abb. 18 . Komplexe Fraktur: C3.3 nach AO (44 Jahre, männlich). a u. b präoperative Röntgenaufnahmen in 2 Ebenen. c u. d Präoperatives CT. e. u.f Primäre Stabilisierung mit Fixateur externe. $\mathbf{g}$ u. h Postoperative Röntgenaufnahmen in 2 Ebenen nach dorsaler und palmarer winkelstabiler Plattenosteosynthese.

gen des R. superficialis des N. radialis zu befürchten und zwar in höherem Maße bei der Osteosynthesematerialentfernung als bei der Einbringung, da Narbenbildung ein Ausweichen des Nervenasts verhindert kann.

\section{Komplikationen}

Die wohl häufigsten Komplikationen sind nach wie vor die sekundäre Dislokation und die in Fehlstellung verheilte distale Radiusfraktur. Hier wird dann in Abhängigkeit der Fehlstellung und der Beschwerdesymptomatik eine Korrekturosteotomie durchgeführt. Fehlstellungen werden bei konservativer Therapie im Gips in bis zu 20\% und bei ausschließlicher Kirschner-Draht-Osteosynthese in unter $10 \%$ der Fälle beobachtet.
Eine weitere relativ häufige Komplikation der distalen Radiusfraktur ist die Entwicklung eines CRPS. Diese wird mit einer Inzidenz von $2-10 \%$ beschrieben. Sehnenabrisse werden bei konservativer (Fragmentkante) und operativer Therapie (Implantat) beobachtet.

\section{Aufklärung}

Der Patient ist zu Behandlungsbeginn über die möglichen Komplikationen aufzuklären. Insbesondere Erwähnung finden sollten:

- Entwicklung eines CRPS

- verbleibende Bewegungseinschränkungen

- Sehnenrupturen, wobei hier vor allem der lange Daumenstrecker und -beuger genannt werden müssen 
Diese Komplikationen sind sowohl bei konservativer als auch bei operativer Frakturbehandlung zu finden.

Alle genannten Osteosyntheseverfahren bergen ebenso wie die konservative Behandlung nach Literaturangaben in etwa das gleiche Risiko der Entwicklung eines CRPS von etwa $3 \%$ der Fälle.

Bei konservativem Therapieversuch muss die Notwendigkeit regelmäßiger radiologischer Nachuntersuchungen in den Vordergrund gestellt werden, da sekundäre Dislokationen je nach Frakturtyp nicht selten sind. Ist eine palmare Plattenosteosynthese geplant, muss über eine Medianusverletzung als mögliche, wenn auch seltene Komplikation gesprochen werden. Über eine sensible Einschränkung der 31/2 radialen Finger hinaus kann eine Schädigung des motorischen Thenarasts auftreten mit Schwäche der Daumenballenmuskulatur.

Häufiger gefährdet ist der R. palmaris. Eine Verletzung des R. palmaris kann eine Hyposensibilität in der Hohlhand zur Folge haben, seltener schmerzhafte Missempfindungen bei Ausbildung eines Neuroms.

Zu nennen ist auch die Entwicklung eines posttraumatischen Karpaltunnelsyndroms, das ebenso nach operativer wie nach konservativer Frakturbehandlung auftreten kann durch narbige Umbauvorgänge des verletzungsbedingten Hämatoms.

Die Implantatentfernung bzw. das geplante Belassen der Implantate (s.o.) sind ebenfalls im Aufklärungsgespräch zu thematisieren.

\section{Prognose}

Das Ergebnis hängt wesentlich vom Frakturtyp ab, wobei zumindest bei jüngeren Patienten ein Zusammenhang zwischen radiologischem Ergebnis und der erreichten Funktion belegt zu sein scheint. Je jünger der Patient ist, umso mehr profitiert er langfristig von einer stufenlosen Wiederherstellung der Gelenkfläche.

Für schlechte Behandlungsergebnisse mitverantwortlich ist häufig eine nicht erkannte oder nicht ausreichend behandelte Begleitverletzung, vor allem durch Entwicklung eines SNAC- bzw. SLAC-Wrist und Instabilitäten im DRUG.

Der Verschleiß bei einer durch Skaphoidpseudarthrose oder skapholunäre Bandruptur bedingten karpalen Instabilität beginnt typischerweise zwischen dem
Tabelle 3

Stadieneinteilung des SLAC- bzw. SNAC-Wrist.

\begin{tabular}{|ll|}
\hline Stadium & Kennzeichen \\
\hline I & Arthrose zwischen proximalem Skaphoidpol und dorsaler Radiuslippe \\
\hline II & Arthrose im gesamten Radioskaphoidalgelenk \\
\hline III & $\begin{array}{l}\text { Arthrose zwischen Lunatum und Kapitatumkopf, später proximoradiale } \\
\text { Migration des Kapitatums durch die skapholunäre Lücke }\end{array}$ \\
\hline
\end{tabular}
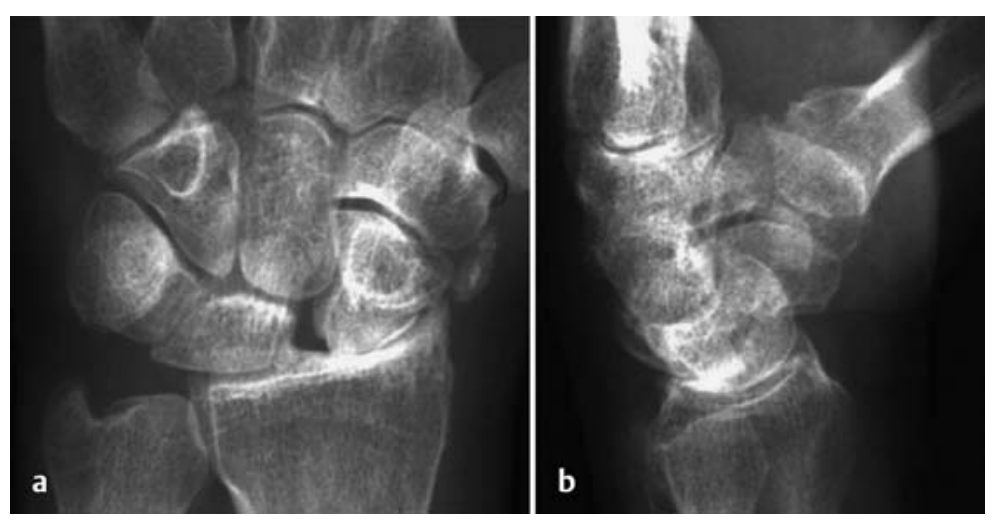

Abb. 19 - SLAC-Wrist Stadium III mit fortgeschrittenem karpalen Kollaps (54 Jahre, weiblich).

proximalen Skaphoidpol und der dorsalen Radiuslippe. Dieser Verschleiß schreitet dann über das gesamte Radioskaphoidalgelenk in Stadien (Tab. 3) fort und kann in eine karpale Panarthrose münden. Ein fortgeschrittenes Arthrosestadium bei statischer skapholunärer Dissoziation ist in Abb. 19 ersichtlich.

Bei älteren Patienten scheint das radiologische Ergebnis nicht zwingend mit dem funktionellen Ergebnis und der Patientenzufriedenheit übereinzustimmen. Bei älteren Patienten mit geringem Anspruch werden auch bei Verbleib schwerwiegender radiologischer Fehlstellungen zufriedenstellende Bewegungsausmaße berichtet.

Komplikationen wie sekundäre Sehnenrupturen etwa der langen Daumenstrecksehne führen ebenfalls zu einer erheblichen Verschlechterung des Outcomes. 


\section{Begutachtung}

Wichtigstes Beurteilungskriterium ist das Bewegungsausmaß im Vergleich zur gesunden Gegenseite.

In der gesetzlichen Unfallversicherung wird eine rentenberechtigende Minderung der Erwerbsfähigkeit (MdE) von $20 \%$ bei einer Bewegungseinschränkung des Handgelenks von insgesamt $80^{\circ}$ anerkannt. Gemessen werden die Handgelenksextensions- und Palmarflexionsbewegungen sowie die Ulnar- und Radialduktionsbewegungen nach der Neutral-0-Methode. Bei Einschränkung der Unteramdrehbewegungen ist je nach deren Schwere die MdE höher zu bewerten.

Eine Bewegungseinschränkung des Handgelenks geringen Grades (z.B. Streckung/Beugung bis $30-0-40^{\circ}$ ) wird mit einem Grad der Behinderung von 0-10 beziffert, eine komplette Handgelenksversteifung in günstiger Stellung mit 20.

$\mathrm{Zu}$ beachten sind radiologische Kriterien wie Stufenbildungen in der Gelenkfläche und Inkongruenzen im distalen Radioulnargelenk, die ein schmerzhaftes Funktionsdefizit erklären können. Arthrotische Veränderungen sollten bei den häufig bereits älteren Versicherten immer im Seitenvergleich beurteilt werden, Gleiches gilt für die Länge der Ulna.

Ob ein posttraumatisch aufgetretenes Karpaltunnelsyndrom (CTS) als Unfallfolge zu werten ist, hängt vom Frakturtyp, dem begleitenden Weichteilschaden und dem zeitlichen Verlauf ab. Primäre Medianusirritationen können bei stark dislozierten Frakturen mit Luxation des Carpus auftreten. Spätere Entwicklungen eines CTS können durch Narbenbildung bedingt sein, bedürfen also einer zum Unfallzeitpunkt dokumentierten Weichteilschädigung oder postoperativen Schwellung. Zumindest muss ein Hämatom im Karpalkanal vorhanden gewesen sein.

Ein unkomplizierter Heilverlauf bei einfacher, wenig dislozierter Radiusfraktur erklärt nicht Medianuskompressionssymptome, die sich erstmals Jahre nach dem Unfall manifestieren. Gleiches gilt für Ringbandstenosen. Auch hier muss eine massive Schwellung der Hohlhand dokumentiert sein, um einen Unfallzusammenhang beweisen zu können.

\section{Prinzipien}

\section{Begutachtungsprinzipien}

- Bewegungsausmaß im Vergleich zur gesunden Gegenseite dokumentieren.

- Auf Gebrauchsspuren der verletzten Hand ist zu achten.

- Ebenso kann eine fehlende Muskelminderung an der verletzten Extremität darauf hinweisen, dass die geklagten Ausfälle mit den tatsächlichen nicht übereinstimmen.

- Bei langjährigem Behandlungsverlauf empfiehlt sich die Auslösung einer Zusatzbegutachtung auf neurologischpsychiatrischem Fachgebiet.

Schwierigkeiten bereitet die Begutachtung des CRPS, da Schmerzen wenig objektivierbar sind. Wichtig ist die Kenntnis der festgelegten CRPS-Kriterien und des stadienhaften Verlaufs, um Rentenbegehren abgrenzen zu können.

Häufig zu beobachten ist die Entwicklung eines Morbus Dupuytren nach Radiusfraktur auch bei Frauen. Dieser betrifft nahezu immer den IV. Strahl, entwickelt sich aber selten zu einem höheren Stadium als Tubiana 0-I. Die Anerkennung als Unfallfolge wird durch nahezu alle Versicherer kritisch gesehen. Da aber ein geringer Ausprägungsgrad der Erkrankung keine Funktionsausfälle bedingt, wird ohnehin selten eine Entschädigung notwendig.

Interessenkonflikt: Die Autoren bestätigen, dass kein Interessenkonflikt vorliegt. 


\section{Zum Weiterlesen und Vertiefen}

1 Arbeitsgemeinschaft der Wissenschaftlichen Medizinischen Fachgesellschaften e.V. [www.awmf.org/leitlinien.html]

2 Arora R, Lutz M, Hennerbichler A et al. Complications following internal fixation of unstable distal radius fracture with a palmar lockingplate. J Orthop Trauma 2007; 21: 316-322

3 Blakeney W. Stabilization and treatment of Colles' fractures in elderly patients. Clin Interv Aging 2010; 5: 337-344

4 Handoll H, Madhok R. Randomized trials do not provide robust evidence for most of the decisions necessary in the management of these fractures. Cochrane Database Syst Rev 2003; 3: CD003209

5 Harden R, Bruehl S, Perez R et al. Validation of proposed diagnostic criteria (the "Budapest criteria") for complex regional pain syndrome. Pain 2010; 150: 268-274

6 Rikli D, Regazzoni R. Fractures of the distal end of the radius: open reduction, internal fixation and early functional after treatment. J Bone Joint Surg 1996; 78-B: 588-592

7 Rikli D, Honigmann P, Morlock MM, Mittlmeier T. Intra-articular pressure measurement in the radiocarpal joint using a novel sensor: in vitro and in vivo results. J Hand Surg 2007; 32 (A): 67-72

\section{Korrespondenzadresse}

Dr. med. Alice Wichelhaus

Chirurgische Universitätsklinik Rostock

Unfall- und Wiederherstellungschirurgie

Schillingallee 35

18055 Rostock

Telefon: 0381/494-6054

Fax: $\quad 0381 / 494-6052$

E-Mail: alice.wichelhaus@med.uni-rostock.de 


\section{Schultergürtel und obere Extremität}

\section{CME-Fragen}

Bei wie viel Prozent der Patienten mit Radiusfrakturen findet sich zum Unfallzeitpunkt eine manifeste Osteoporose?

\section{CME•thieme.de}

\section{CME-Teilnahme}

- Viel Erfolg bei lhrer CME-Teilnahme unter http://cme.thieme.de

- Diese Fortbildungseinheit ist 12 Monate online für eine CME-Teilnahme verfügbar.

- Sollten Sie Fragen zur Online-Teilnahme haben, unter http://cme.thieme.de/hilfe finden Sie eine ausführliche Anleitung.
Eine der folgenden Aussagen zur apparativen Diagnostik bei distaler Radiusfraktur trifft nicht zu. Welche?
A Eine CT-Untersuchung des Handgelenks kann bei Trümmerfrakturen zur OP-Planung hilfreich sein.

B Eine Röntgenuntersuchung der unverletzten Gegenseite ist obsolet.

C Eine MR-Tomografie kann karpale Begleitverletzungen, insbesondere karpale Bandrupturen, aufdecken.

D Die konventionelle Röntgenuntersuchung in 2 Ebenen ermöglicht bei den meisten Radiusfrakturen eine ausreichend genaue Beuteilung der Fraktur.

E Die Arthroskopie gilt als Goldstandard zur Diagnostik einer TFCC-Läsion.
Welches ist kein Instabilitätskriterium einer distalen Radiusfraktur?
A Eine Stufe in der Gelenkfläche von $4 \mathrm{~mm}$.

B Eine Verwerfung im distalen Radioulnargelenk von $2 \mathrm{~mm}$.

C Ein Ulnavorschub von $8 \mathrm{~mm}$.

D Eine Dorsalkippung des distalen gelenktragenden Fragments um $12^{\circ}$.

E Ein radioulnarer Winkel in der a.-p. Röntgenaufnahme von $20^{\circ}$, wenn der Winkel auf der Gegenseite $23^{\circ}$ beträgt.
Welche Aussage zur Notfalltherapie der distalen Radiusfraktur ist zutreffend?
A Eine ulnar I.-gradig offene Fraktur kann wie eine geschlossene Fraktur im Intervall operativ versorgt werden, wenn die Durchspießungswunde mit einem Pflasterverband versorgt wird.

B Wenn die geschlossene Reposition im Aushang nicht ausreichend gelingt, kann das selbe Manöver noch 2-3× wiederholt werden.

C Bei einer komplexen Radiusfraktur mit Luxation im Radiokarpal- und Radioulnargelenk kann die Anlage eines Fixateur externe vor definitiver interner osteosynthetischer Frakturversorgung sinnvoll sein.

D Nach Reposition im Aushang ist bei A2-Frakturen (nach AO-Klassifikation) keine Gipsruhigstellung notwendig.

E Das Aushängen im Mädchenfänger verursacht in der Regel eine Schmerzfreiheit, sodass keine zusätzliche analgetische Therapie oder Bruchspaltanästhesie notwendig ist. 
Wovon ist die Wahl der Therapiemethode bei einer distalen Radiusfraktur nicht abhängig?
A Vom Alter und den Begleiterkrankungen des Patienten.

B Vom Anspruch des Patienten.

C Von der Anzahl der eingenommenen Medikamente.

D Vom Frakturtyp, insbesondere vom Vorliegen einer Gelenkbeteiligung.

E Von Begleitverletzungen am Carpus und Ellbogen.
Welche Aussage zur palmaren winkelstabilen Plattenosteosynthese trifft zu?
A Sie stellt ein Außenseiterverfahren zur Therapie der distalen Radiusfraktur dar.

B Bei diesem Verfahren treten aufgrund der palmaren Plattenlage keine Strecksehnenkomplikationen auf.

C Der operative Zugangsweg liegt ulnar der Handgelenksmitte am Unterarm.

D Bei osteoporotischer Knochenstruktur ist von diesem Verfahren abzuraten.

E Die Platte sollte mittig auf dem Radius proximal der sog. Watershed Line angebracht werden.
Welche Aussage zur Behandlung distaler Radiusfrakturen im Fixateur externe ist korrekt?
A Die Rate der CRPS-Entwicklung liegt bei dieser Behandlungsmethode besonders hoch.

B Bei dorsalen Trümmerzonen kommt es während der Fixateurbehandlung nie zu einem Repositionsverlust.

C Das Anbringen eines Fixateurs verursacht einen erheblichen Weichteilschaden und ist daher bei höhergradigem Weichteilschaden nicht geeignet.

D Vor allem bei komplexem Verletzungsmuster eignet sich der Fixateur zur Primärstabilisierung.

E Bei Ausbehandlung im Fixateur externe ist eine frühfunktionelle Beübung möglich.
Eine der Aussagen zur Aufklärung bei geplanter operativer Versorgung einer distalen Radiusfraktur ist falsch. Welche?
A Bei der palmaren Plattenosteosynthese ist nicht auf die Möglichkeit der Schädigung des N. medianus hinzuweisen, da er weit entfernt vom OP-Gebiet liegt.

B Bei dorsaler Plattenlage können Strecksehnenirritationen auftreten.

C Bei palmarer Plattenlage können Strecksehnenirritationen auftreten.

D Eine Ruptur der Extensor-pollicis-longus-Sehne kann auch bei konservativer Frakturbehandlung beobachtet werden.

E Bei der Behandlung im Fixateur externe muss eine regelmäßige Pinpflege erfolgen, trotzdem können Pininfekte auftreten.
Welche Aussage zur Begutachtung nach distaler Radiusfraktur ist richtig?
A Eine alleinige Einschränkung der Beweglichkeit im Handgelenk für Extension/ Flexion $40-0-60^{\circ}$ bei $60-0-80^{\circ}$ der gesunden Gegenseite bedingt in der gesetzlichen Unfallversicherung eine MdE von $30 \%$.

B Störungen der Umwendbewegungen werden bei der Begutachtung nach distaler Radiusfraktur nicht berücksichtigt.

C Tritt ein Karpaltunnelsyndrom mit einer Latenz von 6 Jahren nach Unfall klinisch in Erscheinung, ist ein Unfallzusammenhang auch bei konservativer Behandlung einer A2-Fraktur wahrscheinlich.

D Ein CRPS hinterlässt in der Regel keinen Dauerschaden.

E Eine Versteifung des Handgelenks in günstiger Stellung ohne weitere Einschränkungen der Handfunktion bedingt eine MdE von $20 \%$.
Was gilt nicht als typische Komplikation bei distaler Radiusfraktur?
A eine posttraumatische Rhizarthrose

B die Entwicklung eines CRPS

C Einschränkungen der Umwendbeweglichkeit bei Mitverletzung des DRUG

D ein sekundärer Korrekturverlust bei dorsaler Trümmerzone

E eine sekundäre Ruptur der langen Daumenstrecksehne 